\title{
Combined Silicon-Phosphorus Fertilization Affects the Biomass and Phytolith Stock of Rice Plants
}

\author{
Zimin $\mathrm{Li}^{1+}$, Fengshan $\mathrm{Guo}^{2 \dagger}$, Jean-Thomas Cornelis ${ }^{3}$, Zhaoliang Song ${ }^{4 *}$, Xudong Wang ${ }^{2}$ \\ and Bruno Delvaux ${ }^{1}$ \\ ${ }^{1}$ Soil Science, Earth and Life Institute, Université catholique de Louvain (UCLouvain), Louvain-la-Neuve, Belgium, ${ }^{2}$ School of \\ Environment and Resources, Zhejiang Agricultural and Forestry University, Lin'an, China, ${ }^{3}$ BIOSE Department, Gembloux \\ Agro-Bio Tech, University of Liege, Gembloux, Belgium, ${ }^{4}$ Institute of the Surface-Earth System Science, Tianjin University, \\ Tianjin, China
}

OPEN ACCESS

Edited by: Julia Cooke,

The Open University (United Kingdom),

United Kingdom

Reviewed by: Jitender Giri,

National Institute of Plant Genome Research (NIPGR), India Timothy J. Gallaher,

Bernice P. Bishop Museum, United States

*Correspondence:

Zhaoliang Song

zhaoliang.song@tju.edu.cn

${ }^{\dagger}$ These authors have contributed equally to this work

Specialty section: This article was submitted to Functional Plant Ecology, a section of the journal

Frontiers in Plant Science

Received: 20 December 2018 Accepted: 17 January 2020

Published: 18 February 2020

Citation:

Li Z, Guo F, Cornelis J-T, Song Z, Wang $X$ and Delvaux B (2020) Combined Silicon-Phosphorus

Fertilization Affects the Biomass and Phytolith Stock of Rice Plants.

Front. Plant Sci. 11:67. doi: 10.3389/fpls.2020.00067
Phytoliths are silica bodies formed in living plant tissues. Once deposited in soils through plant debris, they can readily dissolve and then increase the fluxes of silicon (Si) toward plants and/or watersheds. These fluxes enhance Si ecological services in agricultural and marine ecosystems through their impact on plant health and carbon fixation by diatoms, respectively. Fertilization increases crop biomass through the supply of plant nutrients, and thus may enhance $\mathrm{Si}$ accumulation in plant biomass. Si and phosphorus (P) fertilization enhance rice crop biomass, but their combined impact on Si accumulation in plants is poorly known. Here, we study the impact of combined Si-P fertilization on the production of phytoliths in rice plants. The combination of the respective supplies of $0.52 \mathrm{~g}$ $\mathrm{Si} \mathrm{kg}{ }^{-1}$ and $0.20 \mathrm{~g} \mathrm{P} \mathrm{kg}^{-1}$ generated the largest increase in plant shoot biomass (leaf, flag leaf, stem, and sheath), resulting in a 1.3-fold increase compared the control group. Applying combined Si-P fertilizer did not affect the content of organic carbon (OC) in phytoliths. However, it increased plant available Si in soil, plant phytolith content and its total stock (mg phytolith pot $^{-1}$ ) in dry plant matter, leading to the increase of the total amount of $\mathrm{OC}$ within plants. In addition, $\mathrm{P}$ supply increased rice biomass and grain yield. Through these positive effects, combined Si-P fertilization may thus address agronomic (e.g., sustainable ecosystem development) and environmental (e.g., climate change) issues through the increase in crop yield and phytolith production as well as the promotion of Si ecological services and OC accumulation within phytoliths.

Keywords: phytolith, crop yield, silicon-phosphorus fertilization, rice, silicon cycle

\section{INTRODUCTION}

Amorphous biogenic silica $\left(\mathrm{SiO}_{2} \cdot \mathrm{nH}_{2} \mathrm{O}\right)$ can accumulate in living plant tissues during their growth and development (Conley, 2002; Piperno, 2006). These silica bodies, named phytoliths, are released into the soil after the decomposition of litter and plant residues (Smithson, 1956; Alexandre et al., 1997; Fraysse et al., 2006). Depending on their chemical composition and structure, phytoliths can accumulate in soils and sediments over centuries or millennia, or dissolve and then contribute to the 
pool of aqueous monosilicic acid (dissolved silicon: DSi), which is available for plant root uptake (Bartoli, 1985; Meunier et al., 1999; Fraysse et al., 2009; Struyf et al., 2010; Cornelis and Delvaux, 2016). The elemental composition of phytoliths is influenced by plant species and phytolith morphology (Bartoli and Wilding, 1980; Li et al., 2014; Nguyen et al., 2014). Organic carbon (OC) (0.2-6\%) can be associated with phytoliths (Bartoli, 1985; Parr and Sullivan, 2005; Parr et al., 2010; Zuo and Lü, 2011; Li et al., 2013c; Alexandre et al., 2015). In particular, the occlusion of organic carbon (OC) within phytolith (PhytOC), which is formed in plant tissues, has been proposed as a mechanism which traps the photosynthesized molecules within silica bodies (Parr and Sullivan, 2005; Santos et al., 2012; Alexandre et al., 2015; Reyerson et al., 2016). The occurrence of PhytOC has been reported in various studies (Parr and Sullivan, 2005; Parr et al., 2010; Parr and Sullivan, 2011; Song et al., 2012; Song et al., 2013; Li et al., 2013a; Li et al., 2013b; Huang et al., 2014; Song et al., 2015; Guo et al., 2015; Sun et al., 2016; Pan et al., 2017; Qi et al., 2017; Li et al., 2018a). However, the biological processes leading to the occurrence of PhytOC has not been demonstrated. Therefore, OC content in phytoliths varies depending on the extraction procedure (Parr and Sullivan, 2014; Santos and Alexandre, 2017; Song et al., 2016). These variations led to a debate on the scale of OC occlusion within phytoliths, and on the significance of the PhytOC sink for the global C cycle and climate change mitigation (Parr and Sullivan, 2005; Song et al., 2012; Hodson, 2016; Reyerson et al., 2016; Lorenz and Lal, 2018; Crifò and Strömberg, 2019; Ramesh et al., 2019; Song et al., 2016; Santos and Alexandre, 2017). In addition, OC associated with phytoliths might have a non-photosynthetic origin attributed to the uptake of organic molecules from soil (Santos et al., 2012; Alexandre et al., 2015; Reyerson et al., 2016), which could lead to erroneous $\mathrm{C}$ dating using phytoliths (Hodson, 2016). Therefore, the accurate determination of the phytolith OC content must not only completely eliminate external OC, but also keep the phytolith structure intact and the oxidation of $\mathrm{C}$ in the phytolith to a minimum (Parr and Sullivan, 2014). Overoxidation may significantly underestimate phytolith $\mathrm{C}$ sequestration and should be avoided (Parr and Sullivan, 2014).

In any case, whether phytoliths sequester OC or not, the increase in silicon ( $\mathrm{Si}$ ) uptake undoubtedly enhances plant biomass, $\mathrm{Si}$ and phytolith content in plants [a.o. (Li et al., 2018b; Li et al., 2019)]. The amount of OC that could be associated with phytoliths would depend on plant Si accumulation and thus phytolith content (Li et al., 2013c); therefore, suggesting that regulating Si supply might increase phytolith-associated OC in croplands. In this respect, the combination of $\mathrm{Si}$ and phosphorus (P) fertilization may enhance the contents of plant phytolith and OC associated within phytoliths.

This study is how co-fertilization combining $\mathrm{Si}$ and phosphorus $(\mathrm{P})$ can affect Si availability and plant uptake, as well as phytolith formation in rice. Si uptake improves the growth of Si-accumulator cereals such as rice (Savant et al., 1997; Ma et al., 2001; Ma et al., 2006; Liang et al., 2015). Si fertilization can enhance rice resistance to biotic and abiotic stresses (e.g., pests, water and heat stress, disease, etc.) (Liang et al., 2007; Cooke et al., 2016; Cooke and Leishman, 2016; Coskun et al., 2019), and thus promote rice crop yields and Si accumulation (Savant et al., 1997; Ma et al., 2001; Keller et al., 2012). However, $P$ fertilization also plays an important role in improving yields and promoting plant precocity (George et al., 2001; Lambers et al., 2006; Hammond and White, 2008). In paddy soils, Si and $\mathrm{P}$ fertilization could alleviate $\mathrm{P}$ deficiency, increase P uptake by plants (Ma and Takahashi, 1990; Liang et al., 2007; Hu et al., 2018), and enhance plant available Si in soil, hence improving crop yields (Song et al., 2014; Klotzbücher et al., 2015; Carey and Fulweiler, 2016; Li et al., 2019). Furthermore, plant available Si content in soil may increase after P supply. Besides, Si supply can increase P bioavailability in soil through the competition between silicate and phosphate for sorption on $\mathrm{Al}$ and Fe oxide surfaces that bear positive charges (Parfitt, 1989; $\mathrm{Su}$ and Puls, 2003). Combined Si-P fertilization may thus substantially influence $\mathrm{Si}$ and $\mathrm{P}$ biocycling in the soil-plant system, as well as plant phytolith and chemical composition.

Through a pot experiment in controlled conditions, we aim to address three interconnected questions: 1 ) does Si-P fertilization increase rice biomass? 2) does increased biomass promote plant phytolith formation? and 3) does combined Si-P supply impact the amount of OC associated within phytoliths?

\section{MATERIALS AND METHODS}

The pot experiment was carried out at Zhejiang Agricultural and Forestry University, Lin'an, Zhejiang Province, Eastern China $\left(29^{\circ} 56^{\prime}-30^{\circ} 27^{\prime} \mathrm{N}, 118^{\circ} 51^{\prime}-119^{\circ} 52^{\prime} \mathrm{E}\right)$. This region is characterized by a mid-subtropical monsoon climate with a mean annual precipitation of $1,500 \mathrm{~mm}$, a mean annual temperature of $15.8^{\circ}$ C, 237 frost-free days, and an annual 1,939 h of sunshine.

\section{Pot Experiment Design and Management}

The soil used was a Cambisol, according to the World Reference Base (WRB) key (IUSS, 2014), sampled from the agricultural station at Zhejiang Agricultural and Forestry University. The soil was air-dried, sieved at $2 \mathrm{~mm}$, and mixed with Si-P fertilizers. The soil physico-chemical properties were as follows ( $\mathrm{Lu}, 2000)$ : $\mathrm{pH}_{\text {water }}=5.34 \pm 0.02$, soil organic matter $=30.26 \pm 4.28 \mathrm{~g} \mathrm{~kg}^{-1}$, available $\mathrm{Si}=155.59 \pm 22.73 \mathrm{mg} \mathrm{kg}^{-1}$, available $\mathrm{P}=113.87 \pm 1.35$ mg kg${ }^{-1}$, available $\mathrm{K}=10.33 \pm 1.11 \mathrm{mg} \mathrm{kg}^{-1}$ and available $\mathrm{N}=$ $87.15 \pm 2.47 \mathrm{mg} \mathrm{kg}^{-1}$ (Guo et al., 2015). The analytical methods were described by Lu (2000). Here, plant available Si was assessed using extracts of $\mathrm{NaOAc}$ and acetic acid. Jiayu 253 was selected as the experimental rice (Oryza sativa) cultivar because of its high yield and wide distribution in Zhejiang province.

The experiment was carried out using three fertilization levels, zero $(0)$, medium $(\mathrm{m})$, and high $(\mathrm{h})$, for $\mathrm{Si}\left(\mathrm{Si}_{0}: 0, \mathrm{Si}_{\mathrm{m}}\right.$ : $\left.0.26, \mathrm{Si}_{\mathrm{h}}: 0.52 \mathrm{~g} \mathrm{SiO}_{2} \mathrm{~kg}^{-1}\right)$ using $\mathrm{Na}_{2} \mathrm{SiO}_{3}$, and $\mathrm{P}\left(\mathrm{P}_{0}: 0, \mathrm{P}_{\mathrm{m}}: 0.2\right.$, $\mathrm{P}_{\mathrm{h}}$ : $\left.0.4 \mathrm{~g} \mathrm{~kg}^{-1}\right)$ using $\mathrm{P}_{2} \mathrm{O}_{5}$. Nine treatments $\left(\mathrm{Si}_{0} \mathrm{P}_{0}, \mathrm{Si}_{0} \mathrm{P}_{\mathrm{m}}, \mathrm{Si}_{0} \mathrm{P}_{\mathrm{h}}\right.$, $\mathrm{Si}_{\mathrm{m}} \mathrm{P}_{0}, \mathrm{Si}_{\mathrm{m}} \mathrm{P}_{\mathrm{m}}, \mathrm{Si}_{\mathrm{m}} \mathrm{P}_{\mathrm{h}}, \mathrm{Si}_{\mathrm{h}} \mathrm{P}_{0}, \mathrm{Si}_{\mathrm{h}} \mathrm{P}_{\mathrm{m}}$, and $\left.\mathrm{Si}_{\mathrm{h}} \mathrm{P}_{\mathrm{h}}\right)$ and five replicates per treatment were set up (Table 1). $\mathrm{N}$ and $\mathrm{K}$ fertilizers were 
TABLE 1 | The pot experimental design, as designed following silicon (Si) and phosphorus $(P)$ levels. Different lowercase letters indicate significant differences among all treatments [least significant difference (LSD) test; $p<0.05, n=5$ ].

\begin{tabular}{|c|c|c|c|c|c|}
\hline Number & Treatments & $\begin{array}{l}\mathrm{SiO}_{2} \text { fertil- } \\
\text { izer quantity } \\
\left(\mathrm{g} \mathrm{kg}^{-1}\right)\end{array}$ & $\begin{array}{l}\text { Si fertil- } \\
\text { izer } \\
\text { levels }\end{array}$ & $\begin{array}{l}\mathrm{P}_{2} \mathrm{O}_{5} \text { fertil- } \\
\text { izer quantity } \\
\left(\mathrm{g} \mathrm{kg}^{-1}\right)\end{array}$ & $\begin{array}{c}\text { Phosphoric } \\
\text { fertilizer } \\
\text { levels }\end{array}$ \\
\hline 1 & $\mathrm{Si}_{0} \mathrm{P}_{0}$ & 0.00 & Low & 0.0 & Low \\
\hline 2 & $\mathrm{Si}_{0} \mathrm{P}_{\mathrm{m}}$ & 0.00 & Low & 0.2 & Medium \\
\hline 3 & $\mathrm{Si}_{0} P_{\mathrm{h}}$ & 0.00 & Low & 0.4 & High \\
\hline 4 & $\mathrm{Si}_{\mathrm{m}} \mathrm{P}_{0}$ & 0.26 & Medium & 0.0 & Low \\
\hline 5 & $\mathrm{Si}_{\mathrm{m}} \mathrm{P}_{\mathrm{m}}$ & 0.26 & Medium & 0.2 & Medium \\
\hline 6 & $\mathrm{Si}_{\mathrm{m}} \mathrm{P}_{\mathrm{h}}$ & 0.26 & Medium & 0.4 & High \\
\hline 7 & $\mathrm{Si}_{h} \mathrm{P}_{0}$ & 0.52 & High & 0.0 & Low \\
\hline 8 & $\mathrm{Si}_{h} \mathrm{P}_{\mathrm{m}}$ & 0.52 & High & 0.2 & Medium \\
\hline 9 & $\mathrm{Si}_{h} \mathrm{P}_{\mathrm{h}}$ & 0.52 & High & 0.4 & High \\
\hline
\end{tabular}

applied in all treatments as, respectively, urea ammonium nitrate $\left(0.20 \mathrm{~g} \mathrm{~N} \mathrm{~kg}^{-1}\right)$, and $\mathrm{KCl}\left(0.25 \mathrm{~g} \mathrm{~K} \mathrm{~kg}^{-1}\right)$. All fertilizers were added to soil before planting rice. Soil $\mathrm{pH}$ value and available $\mathrm{Si}$ and $\mathrm{P}$ contents under different levels of $\mathrm{Si}$ and $\mathrm{P}$ supply were

TABLE 2 | Soil pH value and available silicon (Si) and phosphorus (P) contents under different levels of Si and P supply *.

\begin{tabular}{|c|c|c|c|c|c|}
\hline \multirow[t]{2}{*}{ Treatments } & \multirow[t]{2}{*}{$\mathrm{pH}$} & Available P & Available Si & Available $\mathbf{N}$ & Available K \\
\hline & & \multicolumn{4}{|c|}{$\mathrm{mg} \mathrm{kg}^{-1}$} \\
\hline $\mathrm{Si}_{0} \mathrm{P}_{0}$ & $5.47 d$ & $10.25 d$ & $102.45 \mathrm{~cd}$ & 103.37c & $10.25 d$ \\
\hline $\mathrm{Si}_{\mathrm{O}} \mathrm{P}_{\mathrm{m}}$ & $5.50 d$ & $14.58 \mathrm{c}$ & $112.23 c$ & $104.19 b c$ & $14.58 \mathrm{c}$ \\
\hline $\mathrm{Si}_{0} P_{h}$ & $5.59 \mathrm{~cd}$ & $17.84 b$ & $110.23 c$ & $107.40 \mathrm{bc}$ & $17.84 b$ \\
\hline $\mathrm{Si}_{\mathrm{m}} \mathrm{P}_{0}$ & $5.67 \mathrm{C}$ & $14.23 c$ & $123.48 \mathrm{bc}$ & 98.81c & $14.23 c$ \\
\hline $\mathrm{Si}_{m} P_{m}$ & $5.71 \mathrm{c}$ & $17.22 \mathrm{~b}$ & $132.79 b$ & $117.87 \mathrm{~b}$ & $17.22 \mathrm{~b}$ \\
\hline $\mathrm{Si}_{\mathrm{m}} P_{\mathrm{h}}$ & 5. $77 \mathrm{c}$ & $19.27 a$ & 133.28b & $127.00 \mathrm{a}$ & $19.27 a$ \\
\hline $\mathrm{Si}_{h} \mathrm{P}_{0}$ & $6.49 a$ & $14.29 c$ & $142.14 a b$ & 98.17c & $14.29 \mathrm{c}$ \\
\hline $\mathrm{Si}_{\mathrm{h}} \mathrm{P}_{\mathrm{m}}$ & $6.22 b$ & 18.06ab & $153.83 a$ & $98.75 c$ & 18.06ab \\
\hline $\mathrm{Si}_{h} P_{h}$ & 6.38ab & 20.33a & $155.22 a$ & $111.13 \mathrm{~b}$ & $20.33 a$ \\
\hline
\end{tabular}

*The data were collected from Sun et al., 2015. determined by Sun et al. (2015), as presented in Table 2. Each pot ( $0.24 \mathrm{~m}$ diameter, $0.28 \mathrm{~m}$ height) contained $8.5 \mathrm{~kg}$ air-dried soil and was regularly irrigated using tap water $\left(\mathrm{Si}: 0.36 \mu \mathrm{g} \mathrm{L}^{-1}\right)$ at the same level until rice grain harvesting. After a first irrigation of $500 \mathrm{ml}, 1,000 \mathrm{ml}$ of water were supplied per pot during the whole growing period, once every 2 days. Crop harvesting was done 4 months after planting. The rice plant parts were sampled separately: sheath, leaf, flag leaf, and stem. Plant samples were thoroughly washed with deionized water, and then oven dried at $75^{\circ} \mathrm{C}$ until a constant weight was attained, as equal to dry shoot biomass. Rice grains, including rice husk, were also dried at $75^{\circ} \mathrm{C}$ and weighed.

\section{Plant Chemical Analysis}

Dried plant samples were cut into small pieces by stainless steel scissors for the analysis of $\mathrm{Si}$ and phytolith content. Plant samples were fused with Li-metaborate at $950^{\circ} \mathrm{C}$ and dissolved in nitric acid $\left(\mathrm{HNO}_{3} 4 \%\right)$, prior to molybdenum blues colorimetry to determine Si content ( $\mathrm{Lu}, 2000)$.

Microwave digestion in combination with Walkley-Black digestion was used to isolate the phytoliths from plant material (Walkley and Black, 1934; Parr et al., 2001), in order to remove extraneous organic materials thoroughly (Li et al., 2013c). We first checked the presence of phytoliths by optical microscopy to ensure that all extraneous organic materials had been removed (Li et al., 2013c). Then, we further assess the purity of phytolith extract using the scanning electron microscope (SEM) images and energy-dispersive spectroscopy (EDS) (Figure 1). The phytoliths were then oven dried at $75^{\circ} \mathrm{C}$ for $24 \mathrm{~h}$, cooled and weighed. Phytolith particles were dissolved in $\mathrm{HF} 1 \mathrm{M}$ at $45^{\circ} \mathrm{C}$ during $100 \mathrm{~min}$, so that associated OC could be released in the acidic solution ( $\mathrm{Li}$ et al., 2013c). Associated OC content was determined using the potassium dichromate procedure and the soil standard reference GBW07405, ensuring a relative precision below 5\% (Li et al., 2013c). Using plant dry matter, OC and phytolith contents, we computed $\mathrm{OC}_{\text {phyt }}$ and $\mathrm{OC}_{\mathrm{pdm}}$, as the $\mathrm{OC}$
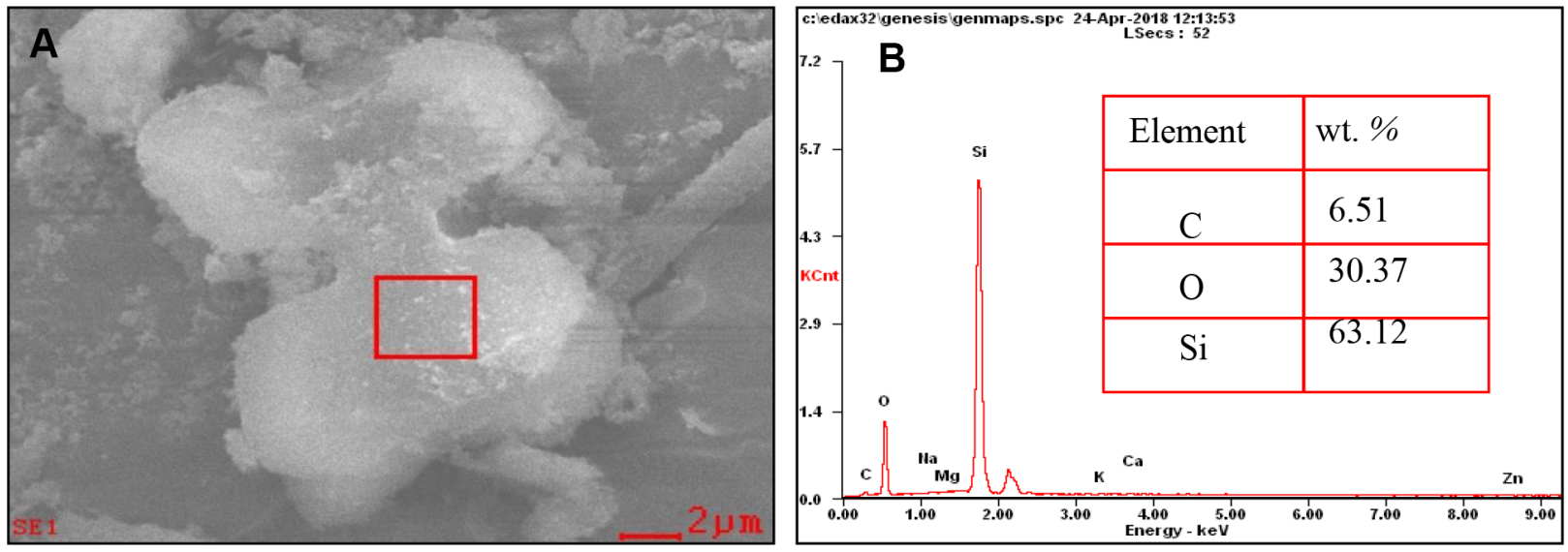

FIGURE 1 | (A) Scanning electron microscope (SEM) image of rice leaf phytolith. (B) Semi-quantitative element concentration (wt. \%, $n=5)$ measured by SEMenergy-dispersive spectroscopy (EDS) of the selected area. 
contents per mass unit of, respectively, phytolith and plant dry matter.

\section{Data Treatment}

Phytolith stock $\left(\mathrm{mg} \operatorname{pot}^{-1}\right)=$ phytolith content $\left(\mathrm{mg} \mathrm{g}^{-1}\right) \times$ biomass of dry plant tissue $\left(\mathrm{g} \mathrm{pot}^{-1}\right)$ where phytolith stock is used to refer to the mass of phytoliths per pot $\left(\mathrm{mg} \mathrm{pot}^{-1}\right)$; phytolith content is used to refer to the mass of phytoliths per gram of dry plant tissue $\left(\mathrm{mg} \mathrm{g}^{-1}\right)$; biomass of dry plant tissue is used to refer to the mass of dry plant tissue per pot $\left(\mathrm{g} \mathrm{pot}^{-1}\right)$.

A two-way analysis of variance of was performed to assess the effects of combined Si-P fertilization levels using SPSS (24.0). Fisher's least significant difference (LSD) test was used to compare the average values of the contents of $\mathrm{SiO}_{2}$, phytolith, $\mathrm{OC}_{\text {phyt }}, \mathrm{OC}_{\mathrm{pdm}}$ in the different plant parts (leaf, flag leaf, sheath, and stem) (at $P<0.05$ level, $\mathrm{n}=5$ ). Origin 8.0 software was used to plot the figures.

\section{RESULTS}

\section{Rice Shoot Biomass and Grain Yield}

The rice shoot biomass $\left(\mathrm{g} \mathrm{pot}^{-1}\right.$ ) significantly varied from 168 in $\mathrm{Si}_{0} \mathrm{P}_{0}$ to 213 in $\mathrm{Si}_{\mathrm{m}} \mathrm{P}_{\mathrm{m}}$ or $\mathrm{Si}_{\mathrm{m}} \mathrm{P}_{\mathrm{h}}$ (Table 3). Among the $\mathrm{Si}_{0}$ treatments, there was a significant increase in shoot biomass between $\mathrm{Si}_{0} \mathrm{P}_{0}$ and $\mathrm{Si}_{0} \mathrm{P}_{\mathrm{h}}$ whereas $\mathrm{Si}_{0} \mathrm{P}_{\mathrm{m}}$ was intermediate between and not significantly different from the other two treatment levels (Table 3). At the given level $\mathrm{P}_{\mathrm{m}}=0.2 \mathrm{~g} \mathrm{~kg}^{-1}$, increasing $\mathrm{Si}$ application rate from $\mathrm{Si}_{0}$ to $\mathrm{Si}_{\mathrm{m}}$ increased the leaf and shoot biomass (Table 3). At the same $\mathrm{P}_{\mathrm{m}}$ level, rice grain yield increased from $\mathrm{Si}_{0}$ to $\mathrm{Si}_{\mathrm{m}}$ and from $\mathrm{Si}_{0}$ to $\mathrm{Si}_{\mathrm{h}}$ (Table 3 ).

\section{Content and Stock of Phytoliths Formed in Rice Plants}

Considering all plant parts, phytolith content significantly varied $(p<0.05)$ from 4.73 to $59.12 \mathrm{mg} \mathrm{g}^{-1}$ (Tables 4-6). At all given levels of $\mathrm{Si}_{0}, \mathrm{Si}_{\mathrm{m}}$, and $\mathrm{Si}_{\mathrm{h}}$, the increase in $\mathrm{P}$ application rate did

TABLE 3 | Effect of silicon-phosphorus (Si-P) levels on biomass in different plant parts and rice dry shoot.

\begin{tabular}{lccccc}
\hline Treatments & Leaf & $\begin{array}{c}\text { Flag } \\
\text { leaf }\end{array}$ & $\begin{array}{c}\text { Stem and } \\
\text { sheath }\end{array}$ & Grains* & $\begin{array}{c}\text { Rice dry } \\
\text { shoot }\end{array}$ \\
\cline { 3 - 6 } & & \multicolumn{5}{c}{ g pot $^{-\mathbf{1}}$} \\
\hline $\mathrm{Si}_{0} \mathrm{P}_{0}$ & $12.40 \mathrm{c}$ & $5.65 \mathrm{bc}$ & $55.62 \mathrm{~b}$ & $102.20 \mathrm{c}$ & $175.88 \mathrm{~b}$ \\
$\mathrm{Si}_{0} \mathrm{P}_{\mathrm{m}}$ & $15.46 \mathrm{a}$ & $6.20 \mathrm{ab}$ & $53.64 \mathrm{~b}$ & $117.05 \mathrm{bc}$ & $192.35 \mathrm{ab}$ \\
$\mathrm{Si}_{0} \mathrm{P}_{\mathrm{h}}$ & $15.76 \mathrm{a}$ & $5.53 \mathrm{~b}$ & $51.53 \mathrm{~b}$ & $130.39 \mathrm{a}$ & $203.21 \mathrm{a}$ \\
$\mathrm{Si}_{\mathrm{m}} \mathrm{P}_{0}$ & $9.98 \mathrm{~d}$ & $4.81 \mathrm{c}$ & $42.80 \mathrm{c}$ & $110.55 \mathrm{c}$ & $171.70 \mathrm{ab}$ \\
$\mathrm{Si}_{m} \mathrm{P}_{\mathrm{m}}$ & $15.22 \mathrm{a}$ & $5.30 \mathrm{bc}$ & $55.81 \mathrm{~b}$ & $136.42 \mathrm{a}$ & $212.92 \mathrm{a}$ \\
$\mathrm{Si}_{m} \mathrm{P}_{h}$ & $13.34 \mathrm{~b}$ & $5.60 \mathrm{bc}$ & $49.36 \mathrm{bc}$ & $141.69 \mathrm{a}$ & $210.99 \mathrm{a}$ \\
$\mathrm{Si}_{h} \mathrm{P}_{0}$ & $12.39 \mathrm{c}$ & $4.72 \mathrm{c}$ & $47.91 \mathrm{bc}$ & $117.60 \mathrm{bc}$ & $182.61 \mathrm{ab}$ \\
$\mathrm{Si}_{h} \mathrm{P}_{\mathrm{m}}$ & $15.59 \mathrm{a}$ & $7.08 \mathrm{a}$ & $59.55 \mathrm{ab}$ & $129.51 \mathrm{ab}$ & $210.07 \mathrm{ab}$ \\
$\mathrm{Si}_{h} \mathrm{P}_{h}$ & $15.15 \mathrm{a}$ & $5.50 \mathrm{bc}$ & $62.28 \mathrm{a}$ & $114.23 \mathrm{bc}$ & $197.15 \mathrm{ab}$ \\
\hline
\end{tabular}

The data of rice organ (leaf, flag leaf, stem and sheath) collected from Sun et al., 2015; Different lowercase letters represent significant differences of rice shoot biomass (Duncan's multiple range test; at $p<0.05$ level, $n=5$ ).

*Grains including rice husk.
TABLE 4 | Contents of $\mathrm{SiO}_{2}$, phytolith, organic carbon (OC) associated with phytolith as expressed per unit mass of phytolith $\left(\mathrm{OC}_{\text {phyt }}\right)$ and of plant dry matter $\left(\mathrm{OC}_{\mathrm{pdm}}\right)$ in different plant parts (leaf, flag leaf, sheath, and stem).

\begin{tabular}{|c|c|c|c|c|c|c|}
\hline \multirow{2}{*}{$\begin{array}{l}\text { Rice } \\
\text { organs }\end{array}$} & \multicolumn{2}{|c|}{ Treatment } & \multirow{2}{*}{$\begin{array}{c}\mathrm{SiO}_{2} \\
\text { content in } \\
\text { plant parts }\end{array}$} & \multirow{2}{*}{$\begin{array}{c}\text { Phytolith } \\
\text { content }\end{array}$} & \multirow{2}{*}{$\begin{array}{l}\text { OC }_{\text {phyt }} \\
\text { ) }\end{array}$} & \multirow[t]{2}{*}{$O C_{p d m}$} \\
\hline & $\begin{array}{c}\mathrm{Si} \\
\text { treatment }\end{array}$ & $\begin{array}{c}\mathbf{P} \\
\text { treatment }\end{array}$ & & & & \\
\hline \multirow[t]{10}{*}{ Leaf } & $\mathrm{Si}_{0}$ & $P_{0}$ & $\begin{array}{c}35.28 \pm \\
5.25 \mathrm{Ba}\end{array}$ & $\begin{array}{l}28.35 \pm \\
3.69 \mathrm{Ca}\end{array}$ & $\begin{array}{l}14.48 \pm \\
1.62 \mathrm{Aa}\end{array}$ & $\begin{array}{c}0.41 \pm \\
0.10 \mathrm{Ba}\end{array}$ \\
\hline & & $P_{m}$ & $\begin{array}{l}34.47 \pm \\
4.21 \mathrm{Ca}\end{array}$ & $\begin{array}{l}31.52 \pm \\
0.70 \mathrm{Ba}\end{array}$ & $\begin{array}{l}14.50 \pm \\
0.41 \mathrm{Aa}\end{array}$ & $\begin{array}{c}0.46 \pm \\
0.02 \mathrm{Ba}\end{array}$ \\
\hline & & $P_{h}$ & $\begin{array}{c}33.46 \pm \\
5.88 \mathrm{Ba}\end{array}$ & $\begin{array}{l}29.39 \pm \\
3.03 \mathrm{Ba}\end{array}$ & $\begin{array}{l}15.16 \pm \\
1.93 \mathrm{Aa}\end{array}$ & $\begin{array}{l}0.45 \pm \\
0.10 \mathrm{Aa}\end{array}$ \\
\hline & $\mathrm{Si}_{\mathrm{m}}$ & $P_{0}$ & $\begin{array}{c}42.35 \pm \\
5.76 \mathrm{Ba}\end{array}$ & $\begin{array}{l}42.37 \pm \\
6.98 \mathrm{Ba}\end{array}$ & $\begin{array}{l}15.48 \pm \\
2.86 \mathrm{Aa}\end{array}$ & $\begin{array}{c}0.67 \pm \\
0.23 \mathrm{ABa}\end{array}$ \\
\hline & & $P_{m}$ & $\begin{array}{c}42.92 \pm \\
0.62 \mathrm{Ba}\end{array}$ & $\begin{array}{c}38.61 \pm \\
4.07 \mathrm{Ba}\end{array}$ & $\begin{array}{l}13.77 \pm \\
0.53 \mathrm{Aa}\end{array}$ & $\begin{array}{c}0.53 \pm \\
0.08 \mathrm{ABa}\end{array}$ \\
\hline & & $P_{h}$ & $\begin{array}{l}44.74 \pm \\
3.95 \mathrm{Aa}\end{array}$ & $\begin{array}{l}34.80 \pm \\
5.08 \mathrm{Ba}\end{array}$ & $\begin{array}{l}13.73 \pm \\
2.47 \mathrm{Aa}\end{array}$ & $\begin{array}{l}0.49 \pm \\
0.16 \mathrm{Aa}\end{array}$ \\
\hline & $\mathrm{Si}_{\mathrm{h}}$ & $P_{0}$ & $\begin{array}{l}67.05 \pm \\
2.84 \mathrm{Aa}\end{array}$ & $\begin{array}{c}59.12 \pm \\
1.39 A a\end{array}$ & $\begin{array}{l}14.71 \pm \\
3.51 \mathrm{Aa}\end{array}$ & $\begin{array}{l}0.87 \pm \\
0.23 \mathrm{Aa}\end{array}$ \\
\hline & & $P_{m}$ & $\begin{array}{l}54.31 \pm \\
5.03 \mathrm{Ab}\end{array}$ & $\begin{array}{l}50.56 \pm \\
4.86 \mathrm{Ab}\end{array}$ & $\begin{array}{l}13.11 \pm \\
1.67 \mathrm{Aa}\end{array}$ & $\begin{array}{c}0.67 \pm \\
0.15 A a b\end{array}$ \\
\hline & & $P_{h}$ & $\begin{array}{c}49.55 \pm \\
0.45 A b\end{array}$ & $\begin{array}{c}43.55 \pm \\
0.30 A c\end{array}$ & $\begin{array}{l}11.16 \pm \\
0.92 \mathrm{Aa}\end{array}$ & $\begin{array}{c}0.49 \pm \\
0.04 \mathrm{Ab}\end{array}$ \\
\hline & & $\begin{array}{l}\text { Mean } \pm \\
\text { s.d. }\end{array}$ & $\begin{array}{l}44.90 \pm \\
10.86 A\end{array}$ & $\begin{array}{l}39.82 \pm \\
10.33 A\end{array}$ & $\begin{array}{c}14.01 \pm \\
1.30 A\end{array}$ & $\begin{array}{l}0.56 \pm \\
0.15 A\end{array}$ \\
\hline \multirow[t]{10}{*}{ Sheath } & $\mathrm{Si}_{0}$ & $P_{0}$ & $\begin{array}{l}21.85 \pm \\
0.95 \mathrm{Ca}\end{array}$ & $\begin{array}{l}19.47 \pm \\
1.49 B b\end{array}$ & $\begin{array}{l}16.74 \pm \\
4.08 \mathrm{Aa}\end{array}$ & $\begin{array}{l}0.33 \pm \\
0.10 \mathrm{Aa}\end{array}$ \\
\hline & & $P_{m}$ & $\begin{array}{c}23.99 \pm \\
0.09 \mathrm{Ba}\end{array}$ & $\begin{array}{l}21.73 \pm \\
3.79 B b\end{array}$ & $\begin{array}{l}18.04 \pm \\
0.10 \mathrm{Aa}\end{array}$ & $\begin{array}{l}0.39 \pm \\
0.07 \mathrm{Aa}\end{array}$ \\
\hline & & $P_{h}$ & $\begin{array}{l}24.31 \pm \\
0.43 \mathrm{Ca}\end{array}$ & $\begin{array}{l}23.44 \pm \\
0.49 \mathrm{Ca}\end{array}$ & $\begin{array}{l}18.17 \pm \\
1.96 \mathrm{Aa}\end{array}$ & $\begin{array}{l}0.43 \pm \\
0.05 \mathrm{Aa}\end{array}$ \\
\hline & $\mathrm{Si}_{\mathrm{m}}$ & $P_{0}$ & $\begin{array}{c}35.85 \pm \\
1.87 \mathrm{Ba}\end{array}$ & $\begin{array}{c}32.00 \pm \\
1.84 \mathrm{Aa}\end{array}$ & $\begin{array}{l}14.79 \pm \\
0.73 \mathrm{Aa}\end{array}$ & $\begin{array}{l}0.47 \pm \\
0.05 \mathrm{Aa}\end{array}$ \\
\hline & & $P_{m}$ & $\begin{array}{c}28.87 \pm \\
1.90 \mathrm{Bb}\end{array}$ & $\begin{array}{l}25.85 \pm \\
2.85 \mathrm{Bb}\end{array}$ & $\begin{array}{l}12.27 \pm \\
3.05 \mathrm{Ba}\end{array}$ & $\begin{array}{l}0.32 \pm \\
0.11 \mathrm{Aa}\end{array}$ \\
\hline & & $P_{h}$ & $\begin{array}{c}35.12 \pm \\
0.20 A a\end{array}$ & $\begin{array}{c}26.88 \pm \\
0.33 \mathrm{Bb}\end{array}$ & $\begin{array}{l}12.77 \pm \\
1.07 \mathrm{Ba}\end{array}$ & $\begin{array}{c}0.34 \pm \\
0.03 \mathrm{Aa}\end{array}$ \\
\hline & $\mathrm{Si}_{\mathrm{h}}$ & $P_{0}$ & $\begin{array}{c}47.82 \pm \\
1.18 \mathrm{Aa}\end{array}$ & $\begin{array}{c}32.80 \pm \\
1.20 A a\end{array}$ & $\begin{array}{c}14.53 \pm \\
4.69 \mathrm{Aa}\end{array}$ & $\begin{array}{l}0.48 \pm \\
0.17 \mathrm{Aa}\end{array}$ \\
\hline & & $P_{m}$ & $\begin{array}{c}37.13 \pm \\
1.53 \mathrm{Ab}\end{array}$ & $\begin{array}{c}35.33 \pm \\
6.33 \mathrm{Aa}\end{array}$ & $\begin{array}{c}15.48 \pm \\
2.11 \mathrm{ABa}\end{array}$ & $\begin{array}{l}0.56 \pm \\
0.17 \mathrm{Aa}\end{array}$ \\
\hline & & $P_{h}$ & $\begin{array}{c}30.93 \pm \\
0.66 \mathrm{Bb}\end{array}$ & $\begin{array}{c}29.79 \pm \\
0.10 A a\end{array}$ & $\begin{array}{l}14.06 \pm \\
1.93 \mathrm{Ba}\end{array}$ & $\begin{array}{c}0.42 \pm \\
0.06 \mathrm{Aa}\end{array}$ \\
\hline & & $\begin{array}{l}\text { Mean } \pm \\
\text { s.d. }\end{array}$ & $\begin{array}{l}31.76 \pm \\
8.20 A B\end{array}$ & $\begin{array}{l}27.54 \pm \\
5.41 \mathrm{AB}\end{array}$ & $\begin{array}{c}13.67 \pm \\
1.72 A\end{array}$ & $\begin{array}{l}0.37 \pm \\
0.06 B\end{array}$ \\
\hline \multirow[t]{8}{*}{ Stem } & $\mathrm{Si}_{\mathrm{O}}$ & $P_{0}$ & $\begin{array}{l}5.86 \pm \\
1.45 \mathrm{Ba}\end{array}$ & $\begin{array}{l}4.73 \pm \\
0.67 \mathrm{Ba}\end{array}$ & $\begin{array}{l}14.34 \pm \\
2.53 \mathrm{Aa}\end{array}$ & $\begin{array}{c}0.07 \pm \\
0.02 \mathrm{Ba}\end{array}$ \\
\hline & & $P_{m}$ & $\begin{array}{l}7.07 \pm \\
2.51 \mathrm{Aa}\end{array}$ & $\begin{array}{l}5.59 \pm \\
1.34 \mathrm{Ba}\end{array}$ & $\begin{array}{l}17.59 \pm \\
0.70 \mathrm{Aa}\end{array}$ & $\begin{array}{c}0.10 \pm \\
0.02 \mathrm{Aa}\end{array}$ \\
\hline & & $P_{h}$ & $\begin{array}{l}8.60 \pm \\
3.73 \mathrm{Aa}\end{array}$ & $\begin{array}{l}7.00 \pm \\
3.11 \mathrm{Ca}\end{array}$ & $\begin{array}{l}17.69 \pm \\
4.49 \mathrm{Aa}\end{array}$ & $\begin{array}{l}0.13 \pm \\
0.09 A a\end{array}$ \\
\hline & $\mathrm{Si}_{\mathrm{m}}$ & $P_{0}$ & $\begin{array}{l}11.85 \pm \\
1.94 \mathrm{Aa}\end{array}$ & $\begin{array}{l}10.41 \pm \\
0.78 \mathrm{Aa}\end{array}$ & $\begin{array}{l}13.50 \pm \\
2.98 \mathrm{Ab}\end{array}$ & $\begin{array}{c}0.14 \pm \\
0.04 \mathrm{Aa}\end{array}$ \\
\hline & & $P_{m}$ & $\begin{array}{l}6.58 \pm \\
1.61 \mathrm{Ab}\end{array}$ & $\begin{array}{l}5.56 \pm \\
1.84 \mathrm{Bb}\end{array}$ & $\begin{array}{l}17.60 \pm \\
0.47 \mathrm{Aa}\end{array}$ & $\begin{array}{c}0.10 \pm \\
0.03 \mathrm{Aab}\end{array}$ \\
\hline & & $P_{h}$ & $\begin{array}{c}7.87 \pm \\
0.51 \mathrm{Ab}\end{array}$ & $\begin{array}{l}5.55 \pm \\
0.40 \mathrm{Bb}\end{array}$ & $\begin{array}{l}11.91 \pm \\
1.60 \mathrm{Ab}\end{array}$ & $\begin{array}{c}0.07 \pm \\
0.01 \mathrm{Ab}\end{array}$ \\
\hline & $\mathrm{Si}_{\mathrm{h}}$ & $P_{0}$ & $\begin{array}{l}11.06 \pm \\
1.75 \mathrm{Aa}\end{array}$ & $\begin{array}{l}10.17 \pm \\
0.85 \mathrm{Aa}\end{array}$ & $\begin{array}{l}12.96 \pm \\
1.35 \mathrm{Aa}\end{array}$ & $\begin{array}{l}0.13 \pm \\
0.02 \mathrm{Aa}\end{array}$ \\
\hline & & $P_{m}$ & $\begin{array}{l}9.83 \pm \\
2.74 \mathrm{Aa}\end{array}$ & $\begin{array}{l}8.79 \pm \\
0.73 \mathrm{Aa}\end{array}$ & $\begin{array}{c}14.80 \pm \\
4.79 \mathrm{Aa}\end{array}$ & $\begin{array}{c}0.13 \pm \\
0.05 \mathrm{Aa}\end{array}$ \\
\hline
\end{tabular}

(Continued) 
TABLE 4 | Continued

\begin{tabular}{|c|c|c|c|c|c|c|}
\hline \multirow{2}{*}{$\begin{array}{l}\text { Rice } \\
\text { organs }\end{array}$} & \multicolumn{2}{|c|}{ Treatment } & \multirow{2}{*}{$\begin{array}{c}\mathrm{SiO}_{2} \\
\text { content in } \\
\text { plant parts }\end{array}$} & \multirow{2}{*}{$\begin{array}{l}\begin{array}{l}\text { Phytolith } \\
\text { content }\end{array} \\
\text { (mg g }^{-1}\end{array}$} & \multirow{2}{*}{$\begin{array}{l}\mathrm{OC}_{\text {phyt }} \\
\\
\text { ) }\end{array}$} & \multirow[t]{2}{*}{$O C_{p d m}$} \\
\hline & $\begin{array}{c}\mathrm{Si} \\
\text { treatment }\end{array}$ & $\begin{array}{c}\mathbf{P} \\
\text { treatment }\end{array}$ & & & & \\
\hline & & $P_{h}$ & $\begin{array}{l}9.05 \pm \\
1.48 \mathrm{Aa}\end{array}$ & $\begin{array}{l}6.20 \pm \\
0.68 \mathrm{Ab}\end{array}$ & $\begin{array}{l}11.76 \pm \\
1.24 \mathrm{Aa}\end{array}$ & $\begin{array}{l}0.07 \pm \\
0.02 \mathrm{Aa}\end{array}$ \\
\hline & & $\begin{array}{l}\text { Mean } \pm \\
\text { s.d }\end{array}$ & $\begin{array}{l}8.64 \pm \\
2.02 C\end{array}$ & $\begin{array}{l}27.54 \pm \\
5.41 \mathrm{AB}\end{array}$ & $\begin{array}{c}13.67 \pm \\
1.72 A\end{array}$ & $\begin{array}{l}0.37 \pm \\
0.06 \mathrm{~B}\end{array}$ \\
\hline \multirow[t]{10}{*}{$\begin{array}{l}\text { Flag } \\
\text { leaf }\end{array}$} & $\mathrm{Si}_{\mathrm{O}}$ & $P_{0}$ & $\begin{array}{c}23.63 \pm \\
1.86 \mathrm{Cb}\end{array}$ & $\begin{array}{l}19.04 \pm \\
2.35 \mathrm{Ca}\end{array}$ & $\begin{array}{l}14.13 \pm \\
0.68 \mathrm{Aa}\end{array}$ & $\begin{array}{c}0.27 \pm \\
0.05 \mathrm{Ba}\end{array}$ \\
\hline & & $P_{m}$ & $\begin{array}{l}25.09 \pm \\
3.40 \mathrm{Ca}\end{array}$ & $\begin{array}{l}19.72 \pm \\
1.44 \mathrm{Ba}\end{array}$ & $\begin{array}{l}15.57 \pm \\
4.25 \mathrm{Aa}\end{array}$ & $\begin{array}{l}0.31 \pm \\
0.11 \mathrm{Aa}\end{array}$ \\
\hline & & $P_{h}$ & $\begin{array}{l}24.02 \pm \\
2.28 \mathrm{Ca}\end{array}$ & $\begin{array}{l}19.59 \pm \\
3.18 \mathrm{Aa}\end{array}$ & $\begin{array}{l}16.04 \pm \\
0.33 \mathrm{Aa}\end{array}$ & $\begin{array}{l}0.31 \pm \\
0.06 \mathrm{Aa}\end{array}$ \\
\hline & $\mathrm{Si}_{\mathrm{m}}$ & $P_{0}$ & $\begin{array}{c}35.21 \pm \\
0.33 \mathrm{Ba}\end{array}$ & $\begin{array}{c}32.00 \pm \\
0.69 \mathrm{Ba}\end{array}$ & $\begin{array}{l}12.02 \pm \\
1.30 \mathrm{Ab}\end{array}$ & $\begin{array}{c}0.39 \pm \\
0.05 \mathrm{ABa}\end{array}$ \\
\hline & & $P_{m}$ & $\begin{array}{l}26.02 \pm \\
3.66 \mathrm{Bb}\end{array}$ & $\begin{array}{c}28.53 \pm \\
4.15 \mathrm{Aa}\end{array}$ & $\begin{array}{l}11.38 \pm \\
0.74 \mathrm{Ab}\end{array}$ & $\begin{array}{l}0.33 \pm \\
0.07 \mathrm{Aa}\end{array}$ \\
\hline & & $P_{h}$ & $\begin{array}{l}37.20 \pm \\
4.46 \mathrm{Aa}\end{array}$ & $\begin{array}{l}27.48 \pm \\
6.25 \mathrm{Aa}\end{array}$ & $\begin{array}{l}15.23 \pm \\
1.14 \mathrm{Aa}\end{array}$ & $\begin{array}{l}0.42 \pm \\
0.13 \mathrm{Aa}\end{array}$ \\
\hline & $\mathrm{Si}_{\mathrm{h}}$ & $P_{0}$ & $\begin{array}{l}48.16 \pm \\
6.87 \mathrm{Aa}\end{array}$ & $\begin{array}{l}42.08 \pm \\
4.89 A a\end{array}$ & $\begin{array}{l}13.34 \pm \\
3.14 \mathrm{Aa}\end{array}$ & $\begin{array}{l}0.57 \pm \\
0.20 \mathrm{Aa}\end{array}$ \\
\hline & & $P_{m}$ & $\begin{array}{l}35.88 \pm \\
5.59 A b\end{array}$ & $\begin{array}{l}32.00 \pm \\
5.26 \mathrm{Ab}\end{array}$ & $\begin{array}{l}11.74 \pm \\
0.17 \mathrm{Aa}\end{array}$ & $\begin{array}{l}0.38 \pm \\
0.07 \mathrm{Aa}\end{array}$ \\
\hline & & $P_{h}$ & $\begin{array}{c}31.14 \pm \\
1.48 \mathrm{Bc}\end{array}$ & $\begin{array}{l}26.33 \pm \\
0.75 \mathrm{Ab}\end{array}$ & $\begin{array}{l}13.54 \pm \\
4.26 \mathrm{Aa}\end{array}$ & $\begin{array}{l}0.36 \pm \\
0.12 \mathrm{Aa}\end{array}$ \\
\hline & & $\begin{array}{l}\text { Mean } \pm \\
\text { s.d. }\end{array}$ & $\begin{array}{l}31.82 \pm \\
8.15 A B\end{array}$ & $\begin{array}{l}27.48 \pm \\
7.45 \mathrm{AB}\end{array}$ & $\begin{array}{c}15.21 \pm \\
2.11 \mathrm{~A}\end{array}$ & $\begin{array}{c}0.41 \pm \\
0.09 A B\end{array}$ \\
\hline
\end{tabular}

Different lowercase letters indicate significant differences among the treatments in different $P$ treatments and rice plant parts at a given Si level, respectively [least significant difference (LSD) test; $p<0.05, n=5$ ]. Different uppercase letters indicate significant differences among the treatments in different Si treatments and rice plant parts at a given $P$ level, respectively ( $L S D$ test; $p<0.05, n=5$ ). Uppercase letters of bolded texts indicate significant differences among different plant parts (leaf, flag leaf, sheath, and stem).
TABLE 6 | Two-way analysis of variance (ANOVA) of silicon-phosphorus (Si-P) levels on the rice shoot biomass, stock of phytolith, and $\mathrm{OC}_{\mathrm{pdm}}$ in rice shoot.

\begin{tabular}{lccc}
\hline Total amount $\left(\mathbf{m g ~ p o t}^{\mathbf{- 1}}\right)$ & Main factor & $\boldsymbol{F}$ & $\boldsymbol{p}$ \\
\hline Biomass & Si fertilization & 0.192 & 0.827 \\
& P fertilization & 2.774 & 0.089 \\
\multirow{3}{*}{ Phytolith } & Si $\times$ P fertilization & 0.270 & 0.894 \\
& Si fertilization & 30.343 & 0.000 \\
& P fertilization & 1.920 & 0.202 \\
OC $_{\text {pdm }}$ & Si $\times$ P fertilization & 2.440 & 0.123 \\
& Si fertilization & 41.540 & 0.000 \\
& P fertilization & 3.322 & 0.059 \\
& Si $\times$ P fertilization & 14.340 & 0.000 \\
\hline
\end{tabular}

not significantly increase phytolith content regardless of plant part, while this effect was not true for sheath with a significant increase from $\mathrm{Si}_{0} \mathrm{P}_{0}$ to $\mathrm{Si}_{0} \mathrm{P}_{\mathrm{m}}$ (Table 5). Yet at given levels $\mathrm{P}_{0}, \mathrm{P}_{\mathrm{m}}$, and $\mathrm{P}_{\mathrm{h}}$, the increase in $\mathrm{Si}$ application rate significantly increased phytolith content in all plant parts (Table 5). Phytolith content in leaves was the highest, and varied from 28.36 to $59.12 \mathrm{mg} \mathrm{g}^{-1}$, with an average of $39.82 \mathrm{mg} \mathrm{g}^{-1}$ (Table 4). As compared to the other plant parts, stem phytolith content was the lowest, with an average value of $7.11 \mathrm{mg} \mathrm{g}^{-1}$. Considering all plant parts, the stock of phytolith formed during the experimental period varied significantly from 152.6 to $1,002.7 \mathrm{mg} \mathrm{pot}^{-1}$ (Figure 2). Si-P fertilization increased the stock of phytoliths formed in all plant parts, including plant shoot biomass (Figure 2). At all given levels of $\mathrm{Si}_{0}, \mathrm{Si}_{\mathrm{m}}$, and $\mathrm{Si}_{\mathrm{h}}$, the increase in $\mathrm{P}$ application rate did not significantly increase phytolith stock regardless of plant part, including in plant shoot biomass (Figure 2; Table 5). Yet at given levels $\mathrm{P}_{0}, \mathrm{P}_{\mathrm{m}}$, and $\mathrm{P}_{\mathrm{h}}$, the increase in $\mathrm{Si}$ application rate significantly increased phytolith stock in all plant parts, including plant shoot biomass. The mean phytolith stock was the highest in sheath $\left(758.3 \mathrm{mg} \mathrm{pot}^{-1}\right)$, followed by leaf $(621.0 \mathrm{mg}$

TABLE 5 | Two-way analysis of variance (ANOVA) of silicon-phosphorus (Si-P) levels on the contents of $\mathrm{SiO}_{2}$, phytolith, organic carbon (OC) associated with phytolith as expressed per unit mass of phytolith (OCphyt) and of plant dry matter (OCpdm), as well as the stock of phytolith and $\mathrm{OC}_{\mathrm{pdm}}$ in different plant parts (leaf, flag leaf, sheath, and stem).

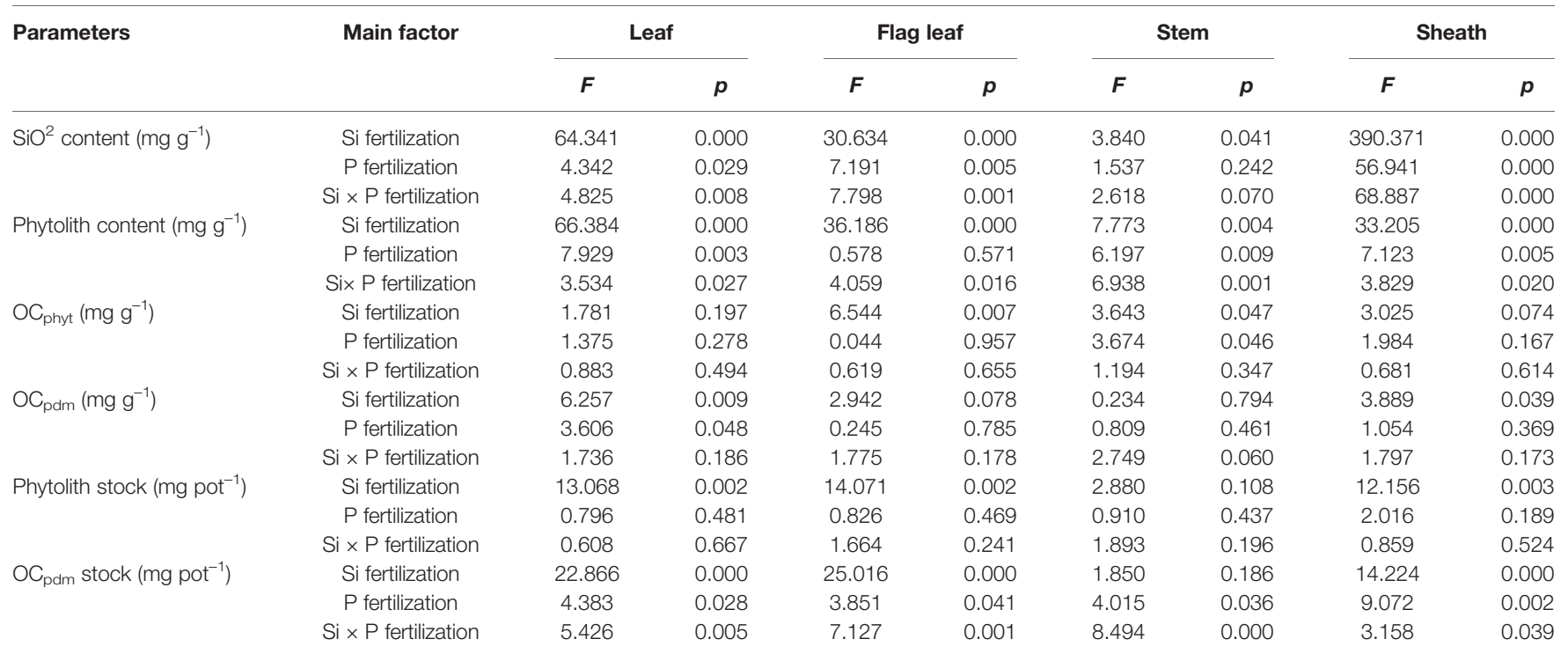




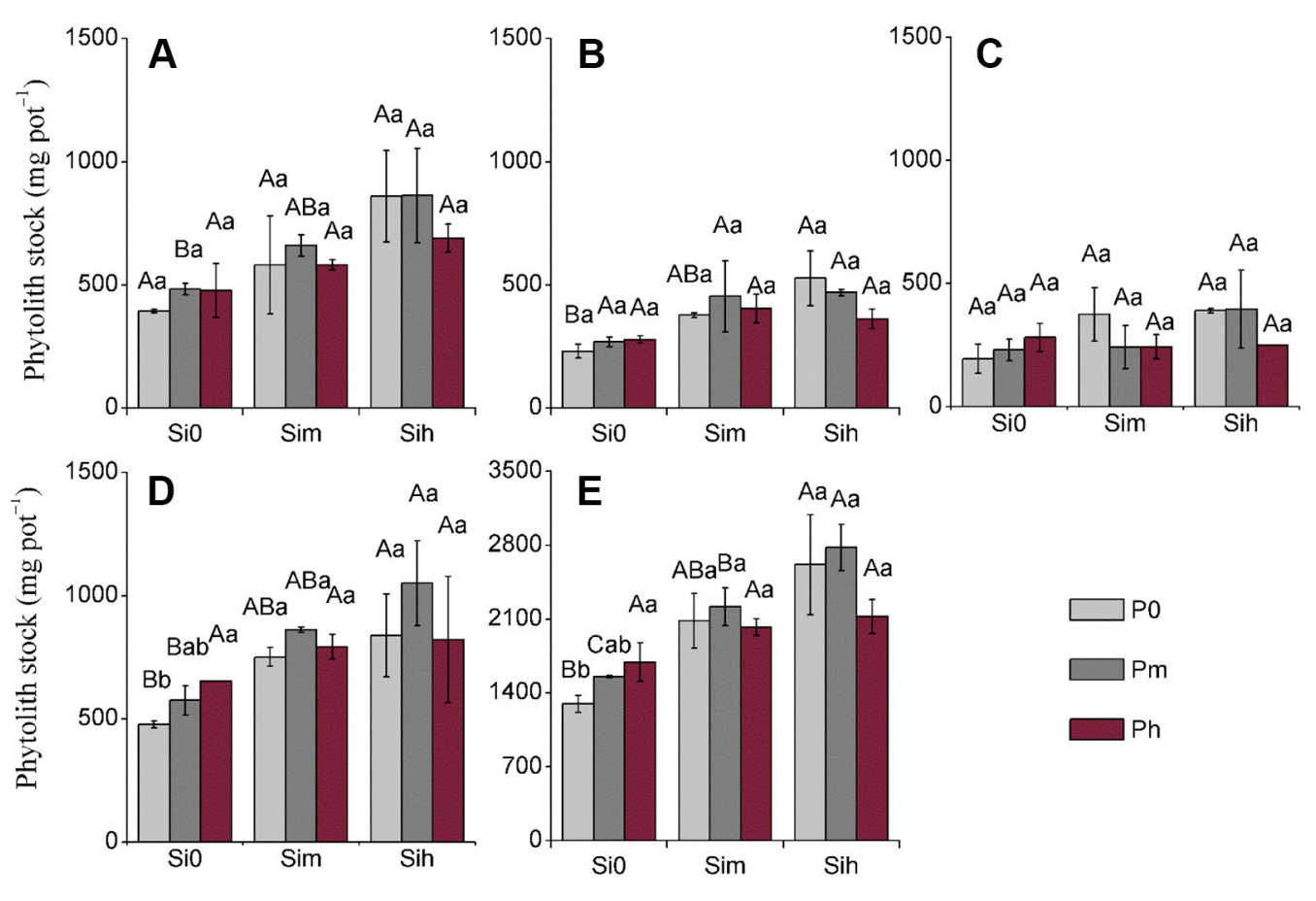

FIGURE 2 | Phytolith stock $\left(\mathrm{mg} \mathrm{pot}^{-1}\right)$ at a two-way analysis of variance of silicon-phosphorus (Si-P) levels in the different plant parts. (A) Leaf; (B) flag leaf; (C): stem; (D): sheath; (E) rice shoot. Error bars represent the standard deviations of the means. Different lowercase letters indicate significant differences among the treatments in different $\mathrm{P}$ treatments and rice plant parts at a given Si level, respectively [least significant difference (LSD) test; $p \leq 0.05, \mathrm{n}=5$ ]. Different uppercase letters indicate significant differences among the treatments in different Si treatments and rice plant parts at a given $\mathrm{P}$ level, respectively (LSD test; $p \leq 0.05, \mathrm{n}=5$ ).

$\operatorname{pot}^{-1}$ ), flag leaf (374.3 $\mathrm{mg}^{-1} \operatorname{pot}^{-1}$ ), and stem (average $289.1 \mathrm{mg}$ pot $\left.^{-1}\right)$. Considering shoot biomass and including rice grains, the stock of phytolith significantly varied from $1,296.6$ to $2,778.6 \mathrm{mg}$ pot $^{-1}$, the latter and maximal value being measured at $\mathrm{Si}_{\mathrm{h}} \mathrm{P}_{\mathrm{m}}$ level (Figure 2).

\section{Organic Carbon Content Associated With Phytoliths Formed in Rice Plants}

Considering all plant parts, $\mathrm{OC}_{\text {phyt }}$ ranged from 11.16 to 18.17 $\mathrm{mg} \mathrm{g}^{-1}$, but did not differ between Si-P treatments and plant parts (Tables 4-6). $\mathrm{OC}_{\text {phyt }}$ content did not vary following $\mathrm{P}$ application irrespective of the $\mathrm{Si}$ supply $\left(\mathrm{Si}_{0}, \mathrm{Si}_{\mathrm{m}}\right.$, and $\left.\mathrm{Si}_{\mathrm{h}}\right)$, while this effect was not true for stem and flag leaves with a significant increase from $\mathrm{Si}_{\mathrm{m}} \mathrm{P}_{0}$ to $\mathrm{Si}_{\mathrm{m}} \mathrm{P}_{\mathrm{m}}$ and $\mathrm{Si}_{\mathrm{m}} \mathrm{P}_{0}$ to $\mathrm{Si}_{\mathrm{m}} \mathrm{P}_{\mathrm{h}}$, respectively (Table 5). At a given level $\mathrm{Si}_{\mathrm{h}}$ in leaf, and a given level $\mathrm{Si}_{\mathrm{m}}$ in stem, the increase in $\mathrm{P}$ application rate significantly decreased their $\mathrm{OC}_{\mathrm{pdm}}$ content (Table 5). At a given level $\mathrm{Si}_{0}$, the increase in $\mathrm{P}$ application rate significantly increased the $\mathrm{OC}_{\mathrm{pdm}}$ stock in all plant parts as well as plant shoot biomass except leaves, while at a given level $\mathrm{Si}_{\mathrm{h}}$ level, the increase in $\mathrm{P}$ application rate significantly decreased the $\mathrm{OC}_{\mathrm{pdm}}$ stock in all plant parts as well as plant shoot biomass except sheath (Table 6 and Figure 3). However, $\mathrm{OC}_{\mathrm{pdm}}$ content and its stock significantly increased with increasing Si application rate due to the increased phytolith content and phytolith stock in all plant parts, respectively (Table 4 and Figure 3).

\section{DISCUSSION}

\section{Effects of Silicon-Phosphorus Supply on Rice Shoot Biomass and Yield}

Our experimental data show that the addition of $\mathrm{P}$ alone increased biomass and grain yield (a significant increase from $\mathrm{Si}_{0} \mathrm{P}_{0} \mathrm{Si}_{0} \mathrm{P}_{\mathrm{m}} \mathrm{Si}_{0} \mathrm{P}_{\mathrm{h}}$ ); but when a combined $\mathrm{Si}-\mathrm{P}$ fertilization were applied there was no significant increase in biomass and yield except that at $\mathrm{Si}_{\mathrm{m}} \mathrm{P}_{\mathrm{m}}$ and $\mathrm{Si}_{\mathrm{m}} \mathrm{P}_{\mathrm{h}}$ (Table 3). This supports the results of previous experiments carried out either in the field (Liu et al., 2014; Liang et al., 2015; Song et al., 2015) or in pots (Agostinho et al., 2017; Liang et al., 1994; Ma and Takahashi, 1990). Si fertilizer supply increased the stock of bioavailable Si that is crucial for sustainable paddy rice yield production (Klotzbücher et al., 2015). Furthermore, once available Si is taken up by plant roots, the accumulation of phytoliths in plant tissues can enhance the efficiency of plant photosynthesis and water use (Meunier et al., 2017), as well as their tolerance to biotic stresses (Epstein, 1994; Cooke and Leishman, 2016; Coskun et al., 2019). On the other hand, P supply likely 

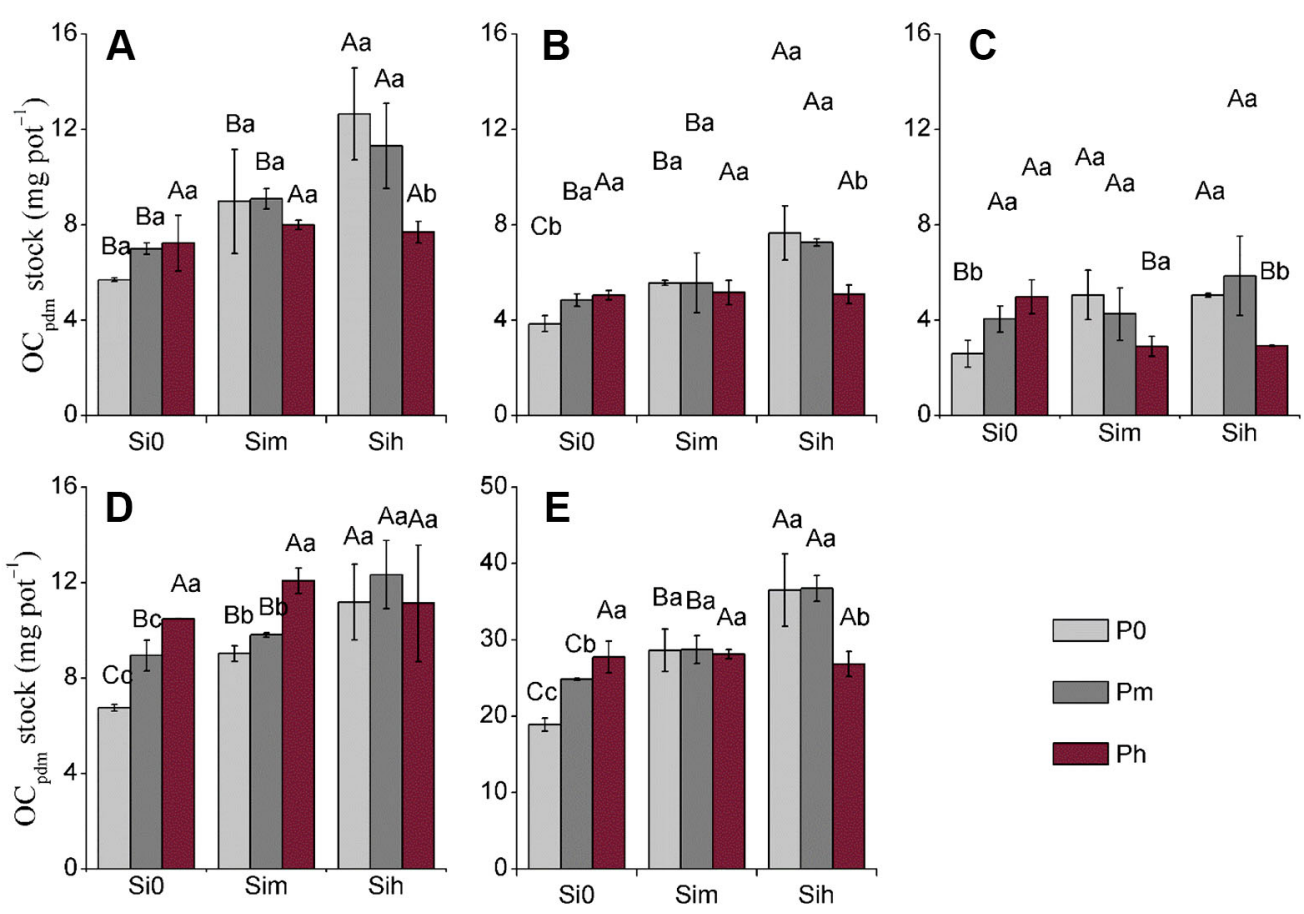

FIGURE 3 | Stock of $\mathrm{OC}_{\mathrm{pdm}}\left(\mathrm{mg} \mathrm{pot}^{-1}\right)$ at two-way analysis of variance of silicon-phosphorus (Si-P) levels in the different plant parts. (A) Leaf; (B) flag leaf; (C): stem; (D): sheath; (E) rich shoot. Error bars represent the standard deviations of the means. Different lowercase letters indicate significant differences among the treatments in different $P$ treatments and rice plant parts at a given Si level, respectively [least significant difference (LSD) test; $p<0.05$ ]. Different uppercase letters indicate significant differences among the treatments in different Si treatments and rice plant parts at a given P level, respectively (LSD test; $p<0.05, n=5)$.

increased plant growth and fecundity as well as root growth (Lambers et al., 2006; Brown et al., 2012). Indeed, low P levels (i.e., $\mathrm{Si}_{\mathrm{m}} \mathrm{P}_{0}$ or $\mathrm{Si}_{\mathrm{h}} \mathrm{P}_{0}$; Table 3 ) did not significantly increase rice biomass regardless of plant part (Tables 2 and 3), confirming that rice growth was clearly limited at low $\mathrm{P}$ supply ( $\mathrm{Ma}$ and Takahashi, 2002; Ma, 2004; Cooke and Leishman, 2016; Agostinho et al., 2017; Hu et al., 2018) even with increasing the addition of Si fertilizer. Excessive inorganic P within rice plant inhibits enzyme reactions, induces abnormal osmotic pressure in plant cell, which further decreases rice growth (Ma and Takahashi, 1990). As reported by Ma and Takahashi (1990), the levels of bioavailable $\mathrm{P}$ and $\mathrm{Si}$ in soil influence plant $\mathrm{P}$ content. At $\mathrm{Si}_{0}$ level, the increase in $\mathrm{P}$ supply did not result in a change of stem, sheath and flag leaf biomass (Table 3) likely because the positive side-effects of $\mathrm{P}$ nutrition were limited at a high $\mathrm{P}$ supply, as mentioned here above. However, these sideeffects may have been enhanced by low Si level. Yet once available $\mathrm{P}$ content increases up to $17.8-20.3 \mathrm{mg} \mathrm{kg}^{-1}$ at $\mathrm{P}_{\mathrm{h}}$ level (Table 2), the increase in bioavailable $\mathrm{Si}$ is beneficial to rice plants by decreasing $\mathrm{P}$ uptake (data not shown; Ma and Takahashi, 1989; Owino-Gerroh and Gascho, 2005; Greger et al., 2018), which, in turn, decreases plant $\mathrm{P}$ content (Ma and Takahashi, 1990). This Si-induced decrease in plant P uptake can also result from the molecular mechanism of down-regulating the expression of $\mathrm{P}$ transporter gene, OsPT6 in rice (Hu et al., 2018). The Si-P interaction thus contributes to increase rice biomass at $\mathrm{Si}_{\mathrm{m}} \mathrm{P}_{\mathrm{m}}, \mathrm{Si}_{\mathrm{m}} \mathrm{P}_{\mathrm{h}}$, and $\mathrm{Si}_{\mathrm{h}} \mathrm{P}_{\mathrm{h}}$ levels (Table 5), suggesting $\mathrm{Si}$ supply may alleviate excessive $\mathrm{P}$ application.

\section{Effects of Silicon-Phosphorus Supply on the Production of Phytoliths}

At a given $\mathrm{P}$ level, $\mathrm{Si}_{2} \mathrm{O}$ content significantly increased with increasing $\mathrm{Si}$ application rate compared to control $\left(\mathrm{Si}_{0}\right)$, regardless of plant part. Thus, the addition of Si fertilizer as monosilicic acid $\left(\mathrm{H}_{4} \mathrm{SiO}_{4}\right)$ taken up by roots resulted in silica accumulation in plant tissues through the formation of phytoliths (Figure 4A). This significant increase was due to the addition of $\mathrm{Si}$ fertilizer that can improve the well-observed increase in plant available $\mathrm{Si}$ in soils (Table 2). The DSi release from highly soluble $\mathrm{Na}_{2} \mathrm{SiO}_{3}$, wollastonite and other Si fertilizers (Haynes et al., 2013; Haynes, 2014; Keeping, 2017; Li et al., 2018b; Li et al., 2019) largely contributed to the pool of bioavailable $\mathrm{Si}$, from which it was taken up by plant roots to accumulate around plant transpiration termini. As expected, $\mathrm{P}$ fertilizer supply did not change the concentration of available $\mathrm{Si}$ in $\mathrm{Si}_{0}$ level (Table 2), and thus of phytolith content, regardless of plant part (Table 4). Interestingly, our data further show that, at given levels $\mathrm{Si}_{\mathrm{m}}$ and $\mathrm{Si}_{\mathrm{h}}$, the increase in $\mathrm{P}$ application rate decreased the formation of phytoliths, but not always significantly, and regardless of plant part, except in flag leaf at $\mathrm{Si}_{\mathrm{h}}$ treatment (Table 4). This trend is in accordance with $\mathrm{Ma}$ and Takahashi (1990) who reported that Si content of rice shoots 

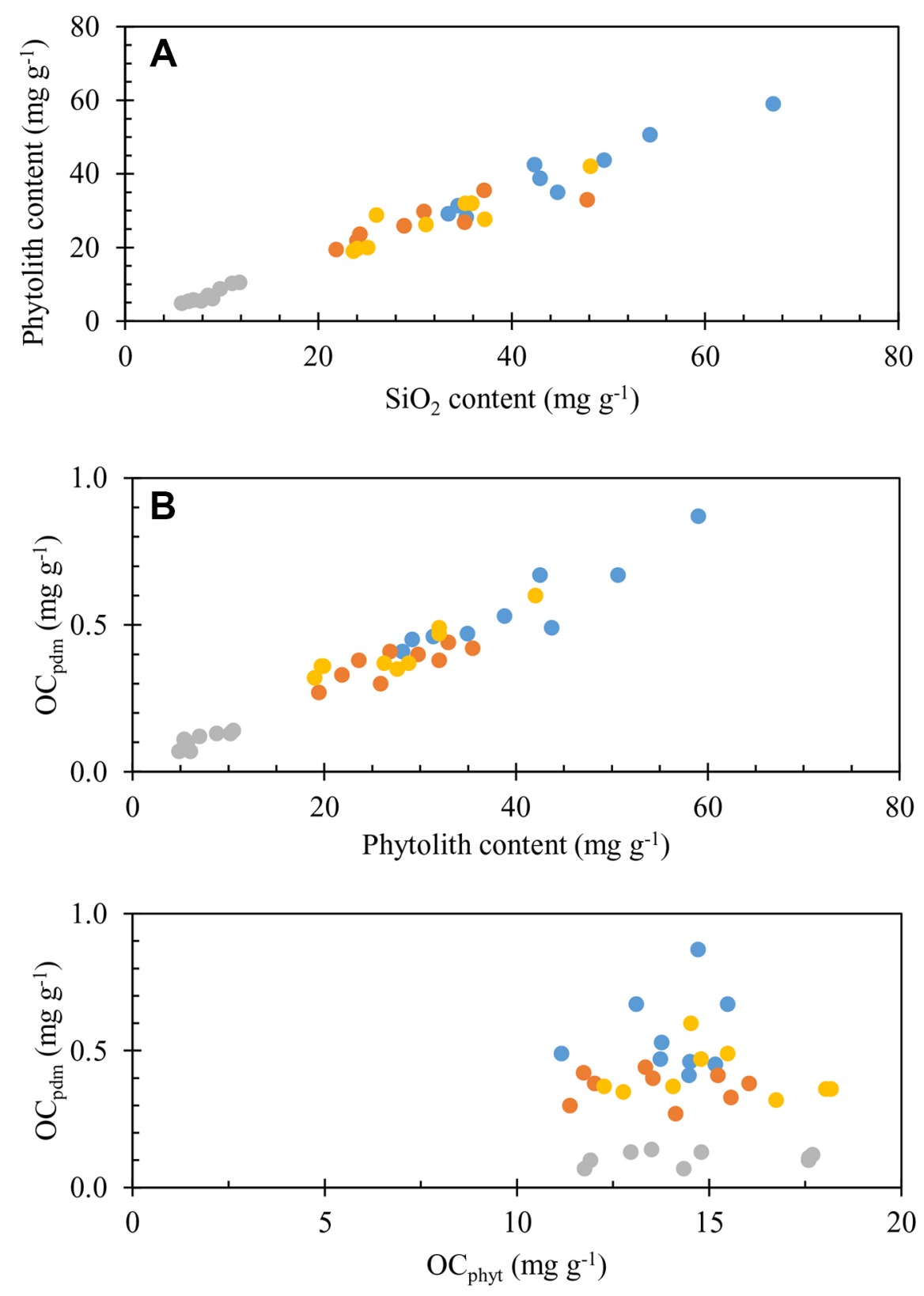

$\bullet$ Leaf $\bullet$ Sheath $\bullet$ Stem $\bullet$ Flag leaf

FIGURE 4 | Plot of: (A) phytolith content of plant parts against $\mathrm{SiO}_{2}$ content considering all silicon-phosphorus (Si-P) treatments (leaf: $y=0.9151 x-1.2668$, $R^{2}=0.9254 P<0.01$; flag leaf: $y=0.8248 x+1.3865, R^{2}=0.8035 P<0.01 ;$ Sheath: $y=0.5457 x+10.337, R^{2}=0.6938 P<0.01 ;$ Stem: $y=1.0171 x-1.6823$, $R^{2}=0.8929 \mathrm{P}<0.01$ ). (B) $O C_{\text {pdm }}$ content of plant parts against phytolith content considering all Si-P treatments (leaf; $\mathrm{y}=0.0134 \mathrm{x}+0.0233, R^{2}=0.8557 \mathrm{P}<0.01$; flag leaf; $y=0.011 x+0.1038, R^{2}=0.8097 P<0.01$; sheath; $y=0.008 x+0.1541 ; R^{2}=0.6845 P<0.01 ;$ stem; $y=0.0121 x+0.0166 ; R^{2}=0.7924 P<0.01$ ). (C) $\mathrm{OC}_{\text {pdm }}$ content of plant parts against $\mathrm{C}$ content of phytoliths $\left(\mathrm{OC}_{\text {phyt }}\right)$ considering all Si-P treatments (leaf; $\mathrm{y}=0.019 \mathrm{x}+0.29, R^{2}=0.0273 \mathrm{P}>0.05$; flag leaf; $y=-0.0079 x+0.5291 ; R^{2}=0.0329 P>0.05 ;$ sheath; $y=-0.003 x+0.4191 ;$ stem; $\left.y=0.0027 x+0.0629 ; R^{2}=0.0491 P>0.05\right)$.

decreased with increasing $\mathrm{P}$ availability in soil (Tables 2 and $\mathbf{3}$ ). As here discussed above, this trend of decreasing Si deposition in plant tissues resulted from dilution caused by increased plant growth following $\mathrm{P}$ application and the molecular mechanism of down-regulating the expression of P transporter gene, OsPT6 in rice (Hu et al., 2018). Since shoot biomass significantly increased following $\mathrm{P}$ addition, our data thus suggest that combined Si-P fertilization contributes to increased Si bioavailability in soil, Si 
root uptake, phytolith formation, and rice plant biomass, which, in turn, increases the stock of phytolith production in plants, while this effect is limited at the high P levels.

\section{Effects of Silicon-Phosphorus Fertilization on Carbon Associated With Rice Phytoliths}

Considering all plant parts (Figure 4), our data suggest that $\mathrm{OC}_{\mathrm{pdm}}$ may be controlled by phytolith accumulation in plant tissues (Figures 4A, B), during which the incorporation of $\mathrm{OC}$ seems to be constant (Figure 4C) and therefore does not influence the $\mathrm{OC}$ content of phytoliths, $\mathrm{OC}_{\text {phyt }}$, in line with previous hypotheses ( $\mathrm{Li}$ et al, 2013c). Evidently, the increase in phytolith stock increases the stock of $\mathrm{OC}_{\mathrm{pdm}}$, i.e., the quantity of OC associated with phytolith in living plant tissues.

Si-P fertilization does not affect $\mathrm{OC}_{\text {phyt }}$ content, regardless of plant part and biomass whereas it affects $\mathrm{OC}_{\mathrm{pdm}}$ (Table 4). SEMenergy dispersive X-ray spectroscopy (EDX) image (Figure 1) illustrates that $\mathrm{OC}$ can be associated with the extracted phytoliths. However, the associated OC levels, irrespective of its source, do not change with the fertilizer treatments. SEMEDX is semi quantitative, and thus, we used this technique not to quantify but to check the OC content as determined chemically. Therefore, we may not conclude about the possible entrapment of OC during polymerization of biogenic amorphous silica as previously proposed (Hodson et al., 1985; Parr and Sullivan, 2005; Zuo and Lü, 2011; Parr and Sullivan, 2014; Alexandre et al., 2015; Alexandre et al., 2016; Reyerson et al., 2016; Hodson, 2016; Song et al., 2016). Similarly, the hypothetical ability of plant phytoliths to occlude OC does not vary depending on the application rate (this study) and type of Si supply: basalt powder (Guo et al., 2015) or slag-based silicate (Song et al., 2015). According to Zhao et al. (2016), increased N supply in degraded grasslands decreased the phytolith content in grass shoots, while significantly increased OC content of their phytoliths. These authors hypothesized that the increase in $\mathrm{OC}_{\text {phyt }}$ was probably caused by improved cell growth, partly enlarged cell volume and decrease in the specific surface area of phytoliths. Similarly, Gallagher et al. (2015) reported, that growing conditions impact the OC content of phytoliths in Sorghum bicolor irrespective of the type and rate of application of inorganic fertilizers. These growth conditions, referring to different nutritive regimes of $\mathrm{N}, \mathrm{P}, \mathrm{K}$, and microelements, affected the plant transpiration stream, and thus $\mathrm{Si}$ accumulation (Gallagher et al., 2015), which in turn, affect the OC content of phytolith (Blackman, 1969; Hodson et al., 1985). In addition to the growth conditions, the nature of plant part or organ might influence the phytolithic OC content through its impact on phytolith morphology and specific surface area (Li et al., 2013c and Li et al., 2014; Table 4).

Although Si-P fertilization did not increase $\mathrm{OC}_{\mathrm{phyt}}$, the application of $\mathrm{Si}$ and $\mathrm{P}$ fertilizer can substantially improve the $\mathrm{OC}_{\mathrm{pdm}}$ content in rice plant through increasing phytolith accumulation (Figures 2 and 3; p $<0.001$ ). Our data further show that the content of phytolithic OC in rice plants mainly depends on Si supply. Indeed, phytolith accumulation in rice plant tissues significantly increased with increasing supply of Si fertilizer. Thus, regulating Si supply promoted the OC content associated within phytolith by increasing phytolith accumulation in plant notably through the increase in biomass production. Consequently, increasing crop productivity could play a crucial role in increasing the stock of phytolithic OC, while the processes explaining OC associated within phytoliths are still debated. Here the largest rice biomass was obtained at $\mathrm{Si}_{\mathrm{h}} \mathrm{P}_{\mathrm{m}}$ level $(\mathrm{Si}=0.52 \mathrm{~g}$ $\mathrm{kg}^{-1} ; \mathrm{P}=0.2 \mathrm{~g} \mathrm{~kg}^{-1}$ ), regardless of plant part (Table 3). The level $\mathrm{Si}_{\mathrm{h}} \mathrm{P}_{\mathrm{m}}$ largely contributed to double the stock of phytolithic OC $\left(\mathrm{mg} \operatorname{pot}^{-1}\right.$ ) from 18.9 at $\mathrm{Si}_{0} \mathrm{P}_{0}$ to 36.8 at $\mathrm{Si}_{\mathrm{h}} \mathrm{P}_{\mathrm{m}}$ (Figure 3E). Another lesson is that $\mathrm{P}$ should not be neglected if rice productivity is to be improved as discussed above. Thus, regulating Si-nutrient supply combined with optimal $\mathrm{P}$ supply is promising to enhance both phytolith formation and associated organic carbon in Siaccumulating plants, as well as crop productivity.

\section{CONCLUSION}

Our experimental results show that i) phytolith concentration increases with increasing Si fertilization, ii) phytolithic OC concentration does not depend on $\mathrm{Si}$ or $\mathrm{P}$ fertilization, iii) as the biomass increases with Si fertilization, the stocks of phytolith and phytolithic OC increase, iv) $\mathrm{P}$ fertilization has no clear impact either on phytolith or phytolithic OC concentration, but increases plant biomass and grain yield. Despite the occurrence of OC associated within phytoliths, we cannot be sure of $\mathrm{OC}$ occlusion within phytoliths. We conclude that the combined Si-P fertilization increases the phytolith stock by increasing the biomass and phytolith content of rice plants. Through these positive effects, combined Si-P fertilization may thus address agronomic (e.g., sustainable ecosystem development) and environmental (e.g., climate change) issues through the increase in crop yield and phytolith production as well as the promotion of $\mathrm{Si}$ ecological services and $\mathrm{OC}$ accumulation within phytoliths.

\section{AUTHOR CONTRIBUTIONS}

We thank Mrs. Linan Liu and Mr. Xiaomin Yang for laboratory assistance (Tianjin University). ZL and FG carried out the experiment, analyzed all data and prepared the draft. XW and $\mathrm{ZS}$ guided the experiment and revised manuscript. J-TC and BD reworked and revised the manuscript. All authors played a significant role in the development of the study and in writing of the manuscript. The submitted version of the manuscript has been read and accepted by all co-authors.

\section{FUNDING}

The work was supported by National Natural Science Foundation of China (41571130042, 41930862 and 41522207) and the State's Key Project of Research and Development Plan of China (2016YFA0601002, 2017YFC0212703). ZL is supported 
by ASP (aspirant)-FNRS of Belgium in 2015-2019 and was also supported by Fonds spécial de recherché of UCLouvain (UCLouvain-FSR) in 2014-2015. The authors declare no competing financial interests.

\section{REFERENCES}

Agostinho, F. B., Tubana, B. S., Martins, M. S., and Datnoff, L. E. (2017). Effect of different silicon sources on yield and silicon uptake of rice grown under varying phosphorus rates. Plants 6 (3), 35. doi: 10.3390/plants6030035

Alexandre, A., Meunier, J.-D., Colin, F., and Koud, J.-M. (1997). Plant impact on the biogeochemical cycle of silicon and related weathering processes. Geochim. Cosmochim. Acta 61, 677-682. doi: 10.1016/S0016-7037(97)00001-X

Alexandre, A., Basile-Doelsch, I., Delhaye, T., Borshneck, D., Mazur, J. C., Reyerson, P., et al. (2015). New highlights of phytolith structure and occluded carbon location: 3-D X-ray microscopy and NanoSIMS results. Biogeosciences 12 (3), 863-873. doi: 10.5194/bg-12-863-2015

Alexandre, A., Balesdent, J., Cazevieille, P., Chevassus-Rosset, C., Signoret, P., Mazur, J. C., et al. (2016). Direct uptake of organically derived carbon by grass roots and allocation in leaves and phytoliths: 13C labeling evidence. Biogeosciences 13, pp.1693-1703. doi: 10.5194/bg-13-1693-2016

Bartoli, F., and Wilding, L. (1980). Dissolution of biogenic opal as a function of its physical and chemical properties. Soil Sci. Soc. America J. 44, 873-878. doi: 10.2136/sssaj1980.03615995004400040043x

Bartoli, F. (1985). Crystallochemistry and surface properties of biogenic opal. Eur. J. Soil Sci. 36, 335-350. doi: 10.1111/j.1365-2389.1985.tb00340.x

Blackman, E. (1969). Observations on the development of the silica cells of the leaf sheath of wheat (Triticum aestivum). Can. J. Bot. 47, 827-838. doi: 10.1139/b69-120

Brown, L. K., George, T. S., Thompson, J. A., Wright, G., Lyon, J., Dupuy, L., et al. (2012). What are the implications of variation in root hair length on tolerance to phosphorus deficiency in combination with water stress in barley (Hordeum vulgare)? Ann. Bot. 110, 319-328. doi: 10.1093/aob/mcs085

Carey, J. C., and Fulweiler, R. W. (2016). Human appropriation of biogenic silicon-the increasing role of agriculture. Funct. Ecol. 30, 1331-1339. doi: $10.1111 / 1365-2435.12544$

Conley, D. J. (2002). Terrestrial ecosystems and the global biogeochemical silica cycle. Global Biogeochem. Cycles 16(4), 68-1. doi: 10.1029/2002GB001894

Cooke, J., and Leishman, M.R.J.F.E. (2016). Consistent alleviation of abiotic stress with silicon addition: a meta-analysis. Functional Ecology 30, 1340-1357. doi: $10.1111 / 1365-2435.12713$

Cooke, J., Degabriel, J. L., and Hartley, S. E. (2016). The functional ecology of plant silicon: geoscience to genes. Funct. Ecol. 30, 1270-1276. doi: 10.1111/13652435.12711

Cornelis, J. T., and Delvaux, B. (2016). Soil processes drive the biological silicon feedback loop. Funct. Ecol. 30(8), 1298-1310. doi: 10.1111/1365-2435.12704

Coskun, D., Deshmukh, R., Sonah, H., Menzies, J. G., Reynolds, O., Ma, J. F., et al. (2019). The controversies of silicon's role in plant biology. New Phytol. 221, no. 1, 67-85. doi: 10.1111/nph. 15343

Crifò, C., and Strömberg, C. A. (2019). Small-scale spatial resolution of the soil phytolith record in a Neotropical rainforest and a dry forest in Costa Rica: applications to the deep-time fossil phytolith record. Palaeogeogr. Palaeoclimatol. Palaeoecol. 537. doi: 10.1016/j.palaeo.2019.03.008

Epstein, E. (1994). The anomaly of silicon in plant biology. Proc. Natl. Acad. Sci. 91, 11-17. doi: 10.1073/pnas.91.1.11

Fraysse, F., Pokrovsky, O. S., Schott, J., and Meunier, J.-D. (2006). Surface properties, solubility and dissolution kinetics of bamboo phytoliths. Geochim. Cosmochim. Acta 70, 1939-1951. doi: 10.1016/j.gca.2005.12.025

Fraysse, F., Pokrovsky, O. S., Schott, J., and Meunier, J.-D. (2009). Surface chemistry and reactivity of plant phytoliths in aqueous solutions. Chem. Geol. 258, 197-206. doi: 10.1016/j.chemgeo.2008.10.003

Gallagher, K. L., Alfonso-Garcia, A., Sanchez, J., Potma, E. O., and Santos, G. M. (2015). Plant growth conditions alter phytolith carbon. Front. Plant Sci. 6, 753. doi: $10.3389 /$ fpls.2015.00753

George, T., Magbanua, R., Roder, W., Van Keer, K., Trébuil, G., and Reoma, V. (2001). Upland rice response to phosphorus fertilization in Asia. Agron. J. 93, 1362-1370. doi: 10.2134/agronj2001.1362

\section{ACKNOWLEDGMENTS}

We thank Mrs. Linan Liu and Mr. Xiaomin Yang for laboratory assistance (Tianjin University).

Greger, M., Landberg, T., and Vaculík, M. (2018). Silicon influences soil availability and accumulation of mineral nutrients in various plant species. Plants 7 (2), 41. doi: 10.3390/plants7020041

Guo, F., Song, Z., Sullivan, L., Wang, H., Liu, X., Wang, X., et al. (2015). Enhancing phytolith carbon sequestration in rice ecosystems through basalt powder amendment. Sci. Bull. 60, 591-597. doi: 10.1007/s11434-015-0729-8

Hammond, J. P., and White, P. J. (2008). Sucrose transport in the phloem: integrating root responses to phosphorus starvation. J. Exp. Bot. 59, 93-109. doi: $10.1093 /$ jxb/erm 221

Haynes, R. J., Belyaeva, O., and Kingston, G. (2013). Evaluation of industrial wastes as sources of fertilizer silicon using chemical extractions and plant uptake. J. Plant Nutr. Soil Sci. 176, 238-248. doi: 10.1002/jpln.201200372

Haynes, R. J. (2014). A contemporary overview of silicon availability in agricultural soils. J. Plant Nutr. Soil Sci. 177, 831-844. doi: 10.1002/jpln.201400202

Hodson, M., Sangster, A., and Parry, D. W. (1985). An ultrastructural study on the developmental phases and silicification of the glumes of Phalaris canariensis L. Ann. Bot. 55, 649-665. doi: 10.1093/oxfordjournals.aob.a086944

Hodson, M. J. (2016). The development of phytoliths in plants and its influence on their chemistry and isotopic composition. Implications for palaeoecology and archaeology. J. Archaeol. Sci. 904 68, 62-69. doi: 10.1016/j.jas.2015.09.002

Hu, A. Y., Che, J., Shao, J. F., Yokosho, K., Zhao, X. Q., Shen, R. F., et al. (2018). Silicon accumulated in the shoots results in down-regulation of phosphorus transporter gene expression and decrease of phosphorus uptake in rice. Plant Soil 423 (1-2), 317-332. doi: 10.1007/s11104-017-3512-6

Huang, Z.-T., Li, Y.-F., Jiang, P.-K., Chang, S. X., Song, Z.-L., Liu, J., et al. (2014). Longterm intensive management increased carbon occluded in phytolith (PhytOC) in bamboo forest soils. Scientific reports 4, 3602. doi: 10.1038/srep03602

IUSS. (2014). World reference base for soil resources 2014 international soil classification system for naming soils and creating legends for soil maps (Rome: FAO).

Keeping, M. G. (2017). Uptake of silicon by sugarcane from applied sources may not reflect plant-available soil silicon and total silicon content of sources. Front. Plant Sci. 8, 760. doi: 10.3389/fpls.2017.00760

Keller, C., Guntzer, F., Barboni, D., Labreuche, J., and Meunier, J.-D. (2012). Impact of agriculture on the Si biogeochemical cycle: input from phytolith studies. Comptes Rendus Geosci. 344, 739-746. doi: 10.1016/j.crte.2012.10.004

Klotzbücher, T., Marxen, A., Vetterlein, D., Schneiker, J., Türke, M., Van Sinh, N., et al. (2015). Plant-available silicon in paddy soils as a key factor for sustainable rice production in Southeast Asia. Basic Appl. Ecol. 16, 665-673. doi: 10.1016/ j.baae.2014.08.002

Lambers, H., Shane, M. W., Cramer, M. D., Pearse, S. J., and Veneklaas, E. J. (2006). Root structure and functioning for efficient acquisition of phosphorus: matching morphological and physiological traits. Ann. Bot. 98, 693-713. doi: $10.1093 / \mathrm{aob} / \mathrm{mcl} 114$

Li, Z., Song, Z., and Jiang, P. (2013a). Biogeochemical sequestration of carbon within phytoliths of wetland plants: a case study of Xixi wetland, China. Chin. Sci. Bull. 58, 2480-2487. doi: 10.1007/s11434-013-5785-3

Li, Z., Song, Z., and Li, B. (2013b). The production and accumulation of phytolithoccluded carbon in Baiyangdian reed wetland of China. Appl. Geochem. 37, 117-124. doi: 10.1016/j.apgeochem.2013.07.012

Li, Z., Song, Z., Parr, J. F., and Wang, H. (2013c). Occluded C in rice phytoliths: implications to biogeochemical carbon sequestration. Plant Soil 370, 615-623. doi: 10.1007/s11104-013-1661-9

Li, Z., Song, Z., and Cornelis, J.-T. (2014). Impact of rice cultivar and organ on elemental composition of phytoliths and the release of bio-available silicon. Front. Plant Sci. 5, 529. doi: 10.3389/fpls.2014.00529

Li, X., Vogeler, I., Schwendenmann, L.Sediments (2018a). Conversion from tussock grassland to pine forest: effect on soil phytoliths and phytolith-occluded carbon (PhytOC). J. Soils Sediments 19 (3), 1260-1271. doi: 10.1007/s11368-018-2160-7

Li, Z., Delvaux, B., Yans, J., Dufour, N., Houben, D., and Cornelis, J.-T. (2018b). Phytolith-rich biochar increases cotton biomass and silicon-mineralomass in a highly weathered soil. J. Plant Nutr. Soil Sci. 181, 537-546. doi: 10.1002/jpln.201800031 
Li, Z., Unzué-Belmonte, D., Cornelis, J.-T., Linden, C. V., Struyf, E., Ronsse, F., et al. (2019). Effects of phytolithic rice-straw biochar, soil buffering capacity and $\mathrm{pH}$ on silicon bioavailability. Plant Soil 438, 187-203. doi: 10.1007/s11104-019-04013-0

Liang, Y. C., Ma, T. S., Li, F. J., and Feng, Y. J. (1994). Silicon availability and response of rice and wheat to silicon in calcareous soils. Communications in Soil Science and Plant Analysis, 25(13-14), 2285-2297.

Liang, Y., Sun, W., Zhu, Y.-G., and Christie, P. (2007). Mechanisms of siliconmediated alleviation of abiotic stresses in higher plants: a review. Environ. Pollut. 147, 422-428. doi: 10.1016/j.envpol.2006.06.008

Liang, Y., Nikolic, M., Bélanger, R., Gong, H., and Song, A. (2015). Silicon in agriculture: From theory to practice (Dordrecht: Springer). doi: 10.1007/978-94-017-9978-2

Liu, X., Li, L., Bian, R., Chen, D., Qu, J., Kibue, G. W., et al. (2014). Effect of biochar amendment on soil-silicon availability and rice uptake. J. Plant Nutr. Soil Sci. 177(1), 91-96. doi: 10.1002/jpln.201200582

Lorenz, K., and Lal, R. (2018). Carbon Sequestration in Grassland Soils in Carbon Sequestration in Agricultural Ecosystems (Springer:Cham), 175-209. doi: 10.1007/978-3-319-92318-5_4

Lu, R. K. (2000). Methods of soil and agro-chemical analysis. China Agricultural Science and Technology Press, Beijing, 127-332.

Ma, J., and Takahashi, E. (1989). Effect of silicic acid on phosphorus uptake by rice plant. Soil Sci. Plant Nutr. 35 (2), 227-234. doi: 10.1080/00380768. 1989.10434755

Ma, J., and Takahashi, E. (1990). Effect of silicon on the growth and phosphorus uptake of rice. Plant Soil 126, 115-119. doi: 10.1007/BF00041376

Ma, J. F., and Takahashi, E. (2002). Soil, fertilizer, and plant silicon research in Japan (Amsterdam: Elsevier). doi: 10.1016/B978-044451166-9/50009-9

Ma, J., Miyake, Y., and Takahashi, E. (2001). Silicon as a beneficial element for crop plants. Stud. Plant Sci. 8, 17-39. doi: 10.1016/S0928-3420(01)80006-9

Ma, J. F., Tamai, K., Yamaji, N., Mitani, N., Konishi, S., Katsuhara, M., et al. (2006). A silicon transporter in rice. Nature 440, 688-691. doi: 10.1038/nature04590

Ma, J. F. (2004). Role of silicon in enhancing the resistance of plants to biotic and abiotic stresses. Soil Sci. Plant Nutr. 50, 11-18. doi: 10.1080/00380768.2004.10408447

Meunier, J. D., Colin, F., and Alarcon, C. (1999). Biogenic silica storage in soils. Geology 27, 835-838. doi: 10.1130/0091-7613(1999)027<0835:BSSIS >2.3.CO;2

Meunier, J. D., Barboni, D., Anwar-Ul-Haq, M., Levard, C., Chaurand, P., Vidal, V., et al. (2017). Effect of phytoliths for mitigating water stress in durum wheat. New Phytol. 215, 229-239. doi: 10.1111/nph.14554

Nguyen, M., Dultz, S., and Guggenberger, G. (2014). Effects of pretreatment and solution chemistry on solubility of rice-straw phytoliths. J. Plant Nutr. Soil Sci. 177 (3), 349-359. doi: 10.1002/jpln.201300056

Owino-Gerroh, C., and Gascho, G. J. (2005). Effect of silicon on low pH soil phosphorus sorption and on uptake and growth of maize. Commun. Soil Sci. Plant Anal. 35 (15-16), 2369-2378. doi: 10.1081/LCSS-200030686

Pan, W., Song, Z., Liu, H., Van Zwieten, L., Li, Y., Yang, X., et al. (2017). The accumulation of phytolith-occluded carbon in soils of different grasslands. J. Soils Sediments 17, 2420-2427. doi: 10.1007/s11368-017-1690-8

Parfitt, R. (1989). Phosphate reactions with natural allophane, ferrihydrite and goethite. Eur. J. Soil Sci. 40, 359-369. doi: 10.1111/j.1365-2389.1989.tb01280.x

Parr, J. F., and Sullivan, L. A. (2005). Soil carbon sequestration in phytoliths. Soil Biol. Biochem. 37, 117-124. doi: 10.1016/j.soilbio.2004.06.013

Parr, J. F., and Sullivan, L. A. (2011). Phytolith occluded carbon and silica variability in wheat cultivars. Plant Soil 342, 165-171. doi: 10.1007/s11104-010-0680-z

Parr, J. F., and Sullivan, L. A. (2014). Comparison of two methods for the isolation of phytolith occluded carbon from plant material. Plant Soil 374 (1-2), 45-53. doi: 10.1007/s11104-013-1847-1

Parr, J. F., Dolic, V., Lancaster, G., and Boyd, W. E. (2001). A microwave digestion method for the extraction of phytoliths from herbarium specimens. Rev. Palaeobot. Palynol. 116, 203-212. doi: 10.1016/S0034-6667(01)00089-6

Parr, J., Sullivan, L., Chen, B., Ye, G., and Zheng, W. (2010). Carbon biosequestration within the phytoliths of economic bamboo species. Global Change Biol. 16, 2661-2667. doi: 10.1111/j.1365-2486.2009.02118.x

Piperno, D. R. (2006). Phytoliths: a comprehensive guide for archaeologists and paleoecologists. Rowman Altamira.

Qi, L., Li, F. Y., Huang, Z., Jiang, P., Baoyin, T., and Wang, H. (2017). Phytolithoccluded organic carbon as a mechanism for long-term carbon sequestration in a typical steppe: the predominant role of belowground productivity. Sci. Total Environ. 577, 413-417. doi: 10.1016/j.scitotenv.2016.10.206
Ramesh, T., Bolan, N. S., Kirkham, M. B., Wijesekara, H., Kanchikerimath, M., Rao, C. S., et al. (2019). "Soil organic carbon dynamics: Impact of land use changes and management practices: A review," in Advances in Agronomy (Academic Press Inc.), 156, 1-107. doi: 10.1016/bs.agron.2019.02.001

Reyerson, P. E., Alexandre, A., Harutyunyan, A., Corbineau, R., Martinez De La Torre, H. A., Badeck, F., et al. (2016). Unambiguous evidence of old soil carbon in grass biosilica particles. Biogeosciences 13, 1269-1286. doi: 10.5194/bg-13-1269-2016

Santos, G. M., and Alexandre, A. (2017). The phytolith carbon sequestration concept: fact or fiction? a comment on "occurrence, turnover and carbon sequestration potential of phytoliths in terrestrial ecosystems by Song et al. Earth-sci. Rev. 164, 251-255. doi: 10.1016/j.earscirev.2016.11.005

Santos, G., Alexandre, A., Southon, J., Treseder, K., Corbineau, R., and Reyerson, P. (2012). Possible source of ancient carbon in phytolith concentrates from harvested grasses. Biogeosciences 9, 1873-1884. doi: 10.5194/bg-9-1873-2012

Savant, N. K., Datnoff, L. E., and Snyder, G. H. (1997). Depletion of plant-available silicon in soils: a possible cause of declining rice yields 1. Commun. Soil Sci. Plant Anal. 28, 1245-1252. doi: 10.1080/00103629709369870

Smithson, F. (1956). Plant opal in soil. Nature 178, 107. doi: 10.1038/178107a0

Song, Z., Liu, H., Si, Y., and Yin, Y. (2012). The production of phytoliths in China's grasslands: implications to the biogeochemical sequestration of atmospheric CO2. Global Change Biol. 18, 3647-3653. doi: 10.1111/gcb.12017

Song, Z., Liu, H., Li, B., and Yang, X. (2013). The production of phytolith-occluded carbon in China's forests: implications to biogeochemical carbon sequestration. Global Change Biol. 19, 2907-2915. doi: 10.1111/gcb.12275

Song, Z., Wang, H., Strong, P. J., and Shan, S. (2014). Increase of available soil silicon by Si-rich manure for sustainable rice production. Agron. Sustain. Dev. 34, 813-819. doi: 10.1007/s13593-013-0202-5

Song, A., Ning, D., Fan, F., Li, Z., Provance-Bowley, M., and Liang, Y. (2015). The potential for carbon bio-sequestration in China's paddy rice (Oryza sativa L.) as impacted by slag-based silicate fertilizer. Sci. Rep. 5, 17354. doi: 10.1038/ srep 17354

Song, Z., McGrouther, K., and Wang, H. (2016). Occurrence, turnover and carbon sequestration potential of phytoliths in terrestrial ecosystems. Earth-Science Reviews 158, 19-30.

Struyf, E., Smis, A., Van Damme, S., Garnier, J., Govers, G., Van Wesemael, B., et al. (2010). Historical land use change has lowered terrestrial silica mobilization. Nat. Commun. 1, 129. doi: 10.1038/ncomms1128

Su, C., and Puls, R. W. (2003). In situ remediation of arsenic in simulated groundwater using zerovalent iron: laboratory column tests on combined effects of phosphate and silicate. Environ. Sci. Technol. 37, 2582-2587. doi: $10.1021 / \mathrm{es} 026351 \mathrm{q}$

Sun, Y., Wang, X., Wang, Y., Pang, W., Lu, R., Ruan, Z., et al. (2015). Soil available nutrients and rice yields with silicon and phosphorus fertilization. J. Zhejiang A\&F Univ. 32, 551-556. doi: 10.11833/j.issn.2095-0756.2015.04.009

Sun, X., Liu, Q., Gu, J., Chen, X., and Zhu, K. (2016). Evaluation of the occluded carbon within husk phytoliths of 35 rice cultivars. Front. Earth Sci. 10(4), 683690. doi: 10.1007/s11707-015-0549-9

Walkley, A., and Black, I. A. (1934). An examination of the Degtjareff method for determining soil organic matter, and a proposed modification of the chromic acid titration method. Soil Sci. 37, 29-38. doi: 10.1097/00010694-193401000-00003

Zhao, Y., Song, Z., Xu, X., Liu, H., Wu, X., Li, Z., et al. (2016). Nitrogen application increases phytolith carbon sequestration in degraded grasslands of North China. Ecol. Res. 31, 117-123. doi: 10.1007/s11284-015-1320-0

Zuo, X., and Lü, H. (2011). Carbon sequestration within millet phytoliths from dry-farming of crops in China. Chin. Sci. Bull. 56, 3451-3456. doi: 10.1007/ s11434-011-4674-x

Conflict of Interest: The authors declare that the research was conducted in the absence of any commercial or financial relationships that could be construed as a potential conflict of interest.

Copyright (c) 2020 Li, Guo, Cornelis, Song, Wang and Delvaux. This is an open-access article distributed under the terms of the Creative Commons Attribution License (CC BY). The use, distribution or reproduction in other forums is permitted, provided the original author(s) and the copyright owner(s) are credited and that the original publication in this journal is cited, in accordance with accepted academic practice. No use, distribution or reproduction is permitted which does not comply with these terms. 